\title{
Optimal Pricing and Production Planning for Subscription-Based Products
}

\author{
Woonghee Tim Huh Soulaymane Kachani_ Ali Sadighian *
}

\begin{abstract}
In this paper, we address the problem of a magazine publishing firm facing stochastic demand over multiple periods. We model the dynamics of customer subscription and retention/attrition. We identify the key decision variables to enhance magazine profitability: production quantity, subscription price and newsstand price.

First, we provide a dynamic programming formulation of the firm's problem and then propose a single-stage reduction which admits a classical newsvendor characterization. For both the finite and infinite horizon cases, we characterize the optimal policy of the firm where the decision variables are one, two or all three of the aforementioned variables. Next, we consider the duopolistic setting, where firms benefit from the overflow of the competitor's unmet demand, and we provide analytic solutions in the case of uniform demand distributions. Finally, we report on computational experiments both in the monopolistic and duopolistic settings and provide managerial insights.
\end{abstract}

\section{Introduction}

\subsection{Motivation and Literature Review}

In this paper, we address the problem of maximizing profit of companies in subscription-based industries. In these industries, such as the magazine publishing industry, there are two main sources of revenue. One is advertisement revenue, and the other is sales of the magazine in the form of either direct sales at the newsstands (spot market) or subscription sales. Information from Audit Bureau of Circulation (2006), Magazine Publishers of America Fact Sheets (2006) and The Magazine Handbook (2006) shows that since 1991, more than $80 \%$ of magazine sales in unit volume have been through subscription as opposed to single-copy sales at the newsstand. This ratio has reached $86 \%$ in 2004. During the same period, subscription revenue as a fraction of total revenue (excluding advertisement) ranged between $67 \%$ and $69 \%$. According to the same sources, magazine publishing companies generated 21.3 billion dollars of advertising revenue in 2004, accounting for $54 \%$ of their total revenue and $48 \%$ of their printed pages.

Our interest in the area of subscription-based products stemmed from a close collaboration, during this past year, between the authors and the managing board of Columbia Journalism Review

\footnotetext{
*School of Engineering and Applied Sciences, Department of Industrial Engineering and Operations Research, Columbia University, New York, NY 10027 \{th2113, sk2267, as2446\}@columbia.edu
} 
(CJR), a leading journalism publication. CJR shared detailed historical operational data and business insights with the authors, which motivated the models and functional forms of the customer dynamics proposed in this paper.

In these subscription-based industries, the key levers to enhance profitability are price (both newsstand price and subscription price) and production quantity. In operations management literature, many mathematical models have been proposed to address pricing and production quantity decisions. Below, we provide a brief literature review of quantity decision models, pricing decision models and joint quantity-pricing models.

An extensive number of papers have studied quantity decision models in inventory literature, dating back to Edgeworth (1888). Prices are given exogenously and the objective is to minimize the expected cost. In many of the multi-period models, solving these problems optimally is known to be difficult even with deterministic and known demand. Zipkin (2000) and Porteus (2002) provide general treatments of inventory models. In this paper, we consider multi-period models with stochastic demand. The most-studied stochastic inventory model is the classic newsvendor problem, where the manager, facing uncertain demand, chooses a stocking quantity to minimize the sum of overage and underage costs. This is a single-period model, and the solution is given by a fractile of the demand distribution. In the analysis of this paper, we use the newsvendor model and its variants.

Another line of research closely related to this paper is dynamic pricing literature. Here, the decision variable is the price over time, while changing the stocking quantity (replenishment or scraping) is usually not a part of the decision. See, for example, Bitran and Mondschein (1995), Gallego and van Ryzin (1994), Smith and Achabal (1998) and Feng and Gallego (1995). There has been recent interest in literature in making the pricing and stocking decisions jointly. Petruzzi and Dada (1999) consider an extension of the newsvendor model where the demand distribution depends on the price set by the newsvendor. In this single-period model, they characterize the optimal solution under fairly general conditions. The multi-period version of this problem has been studied by Federgruen and Heching (1998), where the manager makes both price and inventory replenishment decisions in each period. They consider both the finite and infinite horizon cases. See also Chen and Simchi-Levi (2004a) and Chen and Simchi-Levi (2004b). In this paper, we consider both quantity and pricing decisions. Furthermore, we use two types of prices in each period (newsstand price and subscription price), which differ from single-price-type models that are common in dynamic pricing literature.

While the above discussion is in the monopolistic setting, we also consider competition in our 
paper. Our duopoly model is based on Parlar (1988), where each firm has an initial exogenouslygiven demand allocation and the unmet demand of a firm (if any) will spill over to the other firm. This work was extended by Lippman and McCardle (1997) for more general demand distributions. They prove the existence of an equilibrium in this single-period game and derive the necessary and sufficient conditions for an equilibrium point. We use their result to find the equilibrium of a single-period competition that is analytically equivalent to the multi-period dynamic game of our paper.

We also mention that there is extensive literature on pricing and/or quantity games in a competitive environment, dating back to Cournot (1838 - 1897, English translation by N.T. Bacon) and Bertrand (1883). For example, Kirman and Sobel (1974) consider a game where firms compete on both quantity and price decisions. The demand allocation depends on the price decisions of all firms, and no spill-over effect exists. Bernstein and Federgruen (2004) consider a game where firms compete on price and service level and analyze it using supermodular games. In these papers, the dynamics of competition is different from our model. Furthermore, while they show the existence and the structural properties of the equilibrium, they do not provide closed-form solutions.

\subsection{Contributions and Paper Structure}

Ahn and Olsen (2005) propose a general model for duopolistic competition in subscription-based industries. Competition occurs in two stages: first through quantity and then through subscription prices. They show that there exits a Markov perfect equilibrium under certain technical assumptions. They consider a finite horizon model, and their analysis appeals to the theory of supermodular games introduced by Topkis (1998) and focuses on theoretical existence results.

In this paper, we build on their work. We consider not only the finite horizon case, but also the infinite horizon case. In addition to production quantity and subscription price, we add newsstand price as a decision variable. Furthermore, in our model, we allow subscription prices to change over time. We choose more specific, thus tractable, functional forms of dynamics, which allow us to derive closed-form solutions both in the monopolistic and duopolistic settings, and from which we derive managerial insights. Our ability to extend their work and derive closed-form solutions stems from a one-stage reduction of the problem that is analytically equivalent to the classic newsvendor problem.

The paper is organized as follows. In Section 2, we describe our model in the monopolistic setting and propose an equivalent reformulation as a one-stage problem. In Section 3, assuming a specific behavior for subscription and retention dynamics, we derive closed-form solutions that bring 
insight to the problem. We first analyze the model where the production quantity is the sole decision variable. We then extend our results to the case where subscription price is also a decision variable and further extend it to the case where newsstand price is a third decision variable. In Section 4 , we extend our model to the duopolistic setting, where firms compete over production quantity and subscription prices. The firms interact through the fact that the demand that is unmet by one firm overflows to the other. The results from the monopolistic case carry over to the duopolistic case. We compute the equilibrium production quantity and subscription price decisions in closed-form. Based on the cost structure of the firms and the loyalty of their customers, different types of equilibria emerge and are characterized. We show that a tâtonnement scheme converges to the equilibrium point of the competition which is analytically computable. This result indicates that even if the firms do not consider the full range of their choices and just act with the best response to their competitor's choice of production quantity, they will eventually converge to the equilibrium policy. In Section 5, we perform a comprehensive computational analysis. For instance, to characterize market separation, we examine customers' tendency to switch magazines. We observe that when the cost structures of the two firms are very different, a firm might produce more than the upper bound of its initial stochastic demand in anticipation of the overflow of unsatisfied demand from the other firm. On the other hand, in the case where the firms are not radically different, they both produce within the support of their original stochastic demand. Finally, in Section 6, we provide conclusions and future steps in the analysis of this problem.

\section{Model Definition and the Single-Stage Reduction}

In Section 2.1, we formally describe our model. In each period, a firm produces a certain number of magazines and sells them either through the newsstand or to the subscribers. We formulate this problem as a multi-period dynamic program (DP) in Section 2.2. In Section 2.3, we provide an alternative representation of this dynamic program, which is analytically equivalent to a series of single-stage problems.

\subsection{Model Definition}

We consider a firm in a multi-period setting, where the firm makes a production decision as well as pricing decisions in each period. There are two kinds of demands that the firm faces: (i) the demand at the newsstand and (ii) demand from the subscribers, which we model as follows. In each period, a stochastic demand is realized at the newsstand and this demand is satisfied to the extent possible based on the stocking quantity of the magazines at the newsstand. Then, a portion 
of the satisfied demand subscribes to the magazine. We assume that the only way to subscribe the magazine is to purchase a copy of the magazine and become a subscriber. At the end of each period, some subscribers cancel their subscription, decreasing the number of total subscribers for the following period.

The objective of the firm is to maximize the expected discounted profit over the planning horizon. The revenue consists of three components: direct sales at the newsstand, subscription and advertisement. The only cost we model is the variable cost of production. The revenue and cost functions are described later in this section.

We index periods forward by $t=1,2, \ldots, T$. (Here, we allow the case where $T$ is either finite or infinite.) At the beginning of each period $t$, let $N^{t}$ represent the number of subscribers, which the firm observes. Then, the firm publishes $N^{t}+q^{t}$ copies of the magazine, where $q^{t}$ is the number of copies sent to the newsstand, and $N^{t}$ copies are sent to the subscribers. We denote the stochastic newsstand demand by $D^{t}$. (We allow the distribution of $D^{t}$ to be price-sensitive and dependent on the newsstand price.) Let $\Phi^{t}=\min \left\{q^{t}, D^{t}\right\}$ be a random variable representing the quantity of newsstand demand satisfied. The number of new subscribers is given by $\Delta^{t} \cdot \Phi^{t}$, where $\Delta^{t} \in[0,1]$ represents a random fraction. Let $\delta^{t}=E\left[\Delta^{t}\right]$, which we call the conversion rate. We can interpret $\delta^{t}$ at a macro level as an expected ratio of new subscribers to the satisfied newsstand demand and at a micro level as a probability that an individual will subscribe to the magazine after purchasing a copy from the newsstand.

We now give a recursive formula for the number of subscribers at the beginning of the next period $t+1$. We classify subscribers at the beginning of period $t$ based on when they started their subscription. Let $N^{t k}$ be the number of subscribers at the beginning of period $t$ who started subscription in period $k$, where $k<t$. Thus, $N^{t}=\sum_{k=1}^{t-1} N^{t k}$. Let $\mathbf{N}^{t}=\left(N^{t k} \mid k=1,2, \ldots, t-1\right)$. For each $k$ and $t$ satisfying $k<t, B^{t k} \in[0,1]$ denotes a random fraction representing the retention rate, the percentage of $N^{t k}$ who keep their subscription at the end of period $t$. We assume that the retention rates of new subscribers in period $k$ are such that the sequence of random variables $\left(B^{t k} \mid t=k+1, k+2, \ldots\right)$ are independent and identically distributed. Let $\beta^{k}=E\left[B^{t k}\right]$ where $t=k+1$, which we refer to as the retention rate. Then, for $t \geq 1$,

$$
N^{t+1}=\Delta^{t} \cdot \Phi^{t}+\sum_{k=1}^{t} N^{t+1, k} \quad \text { where } \quad N^{t+1, k}=B^{t k} \cdot N^{t k} \quad \text { for } k=1, \ldots, t .
$$

(Note that $N^{1}$ represents the number of subscribers at the beginning, which is one of the input parameters. Without loss of generality, we assume $N^{1}=0$.) 
Now, we describe the revenue and cost functions in each period. The production cost is linear in the number of magazines produced in each period, where $c \geq 0$ represents the per-unit cost, i.e., the production cost in period $t$ is $c \cdot\left(N^{t}+q^{t}\right)$. Note that at the beginning of period $t$, the value of $N^{t}$ is observed, and thus $c \cdot\left(N^{t}+q^{t}\right)$ is a deterministic quantity. The revenue in each period consists of three parts. The first part comes from direct sales at the newsstand and is given by $p^{t} \cdot \Phi^{t}$, where $p^{t} \geq 0$ represents the newsstand price in period $t$. The second part comes through subscription. We assume that the subscribers pay the subscription price at which they started subscription and that a subscriber's price does not change over time. Let $s^{k} \geq 0$ be the subscription price for the customers who subscribed in period $k$. Then, the subscription revenue in period $t$ is $\sum_{k=1}^{t-1} s^{k} N^{t k}$. The third part of the revenue comes from advertisement. We assume that the advertising revenue is proportional to the number of subscribers, i.e., $m \cdot N^{t}$, where $m \geq 0$ is advertising revenue per subscriber. Therefore, the expected profit of the firm in period $t$ is given by

$$
-c \cdot\left(q^{t}+E\left[N^{t}\right]\right)+p^{t} E\left[\Phi^{t}\right]+\sum_{k=1}^{t-1} s^{k} E\left[N^{t k}\right]+m E\left[N^{t}\right] .
$$

Notice that while the above expression depends on both $q^{t}$ and $p^{t}$, the subscription price $s^{t}$ in period $t$ does not affect the profit generated in that period. We let $\alpha$ denote the discount factor for the profit, where $\alpha \in(0,1)$.

If the planning horizon $T$ is finite, we make the following assumptions regarding the end of the horizon. The value of the firm at the end of the planning horizon is given by a fixed-plus-linear function, $\tilde{v}+N^{T+1} v$, where $\tilde{v} \geq 0$ is the value of the firm regardless of the number of subscribers, $v \geq 0$ is the value of each subscriber and $N^{T+1}$ is the number of subscribers at the end of the planning horizon. The variable component of the salvage value above reflects the fact that the firm is more valuable if it has a larger number of subscribers.

The decision variables in each period $t$ are all or some of the following: (i) the stocking quantity $q^{t}$ at the newsstand, (ii) the subscription price $s^{t}$ and (iii) the newsstand price $p^{t}$. The random variables used in the model are endogenous and controllable by the decision variables; more specifically, demand $D^{t}$ depends on the newsstand price $p^{t}$, the retention fraction $B^{t k}$ depends on the subscription price $s^{k}$, and the conversion fraction $\Delta^{t}$ depends on both prices $s^{t}$ and $p^{t}$. We sometimes make the above dependencies explicit in the notation, e.g., $D^{t}\left(p^{t}\right)$. For any fixed-price decisions, we assume that all of the random variables are independent.

The model described in this section is valid for the monopolistic case, and we extend this model for the duopolistic case later in Section 4. 


\subsection{Dynamic Programming Formulation}

In this section, we present a multi-period dynamic programming formulation for the model described in Section 2.1. We consider the above problem in the following three settings based on the set of levers available to the firm.

- Model Q: Both the newsstand price $p^{t}$ and the subscription price $s^{t}$ are exogenously given, and the firm decides on the newsstand stocking quantity $q^{t}$.

- Model QS: The newsstand price is exogenously given, and the firm decides both the stocking quantity and the subscription price.

- Model QSP: The firm decides all three decision variables, namely the stocking quantity and both the newsstand and subscription prices.

Recall $\mathbf{N}^{t}=\left(N^{t k} \mid k=1,2, \ldots, t-1\right)$ where $N^{t k}$ is the number of subscribers at the beginning of period $t$ who started subscription in period $k$. Define $\mathbf{s}^{t}=\left(s^{k} \mid k=1,2, \ldots, t-1, t\right)$ to be the vector of historical subscription prices. We now present the dynamic programming formulation for the firm. The objective is to maximize $V^{1}\left(\mathbf{s}^{0}, \mathbf{N}^{1}\right)$, where

$$
\begin{array}{rlr}
V^{t}\left(\mathbf{s}^{t-1}, \mathbf{N}^{t}\right) & =\max \quad W^{t}\left(q^{t}, \mathbf{s}^{t}, p^{t}, \mathbf{N}^{t}\right) \quad \text { for } t \leq T & \\
W^{t}\left(q^{t}, \mathbf{s}^{t}, p^{t}, \mathbf{N}^{t}\right) & =g^{t}\left(q^{t}, \mathbf{s}^{t}, p^{t}, \mathbf{N}^{t}\right)+\alpha \cdot V^{t+1}\left(\mathbf{s}^{t}, \mathbf{N}^{t+1}\right) & \text { for } t \leq T \\
V^{T+1}\left(\mathbf{s}^{T}, \mathbf{N}^{T+1}\right) & =\alpha^{T+1} * g^{T+1}\left(\mathbf{N}^{T+1}\right) . &
\end{array}
$$

Above, the maximization is taken over $\left\{q^{t}\right\},\left\{q^{t}, s^{t}\right\}$ or $\left\{q^{t}, s^{t}, p^{t}\right\}$, depending on the application of Model Q, QS or QSP. Furthermore, the single-period expected profit function $g^{t}$ and the evolution of the quantities of interest are defined as follows:

$$
\begin{aligned}
g^{t}\left(q^{t}, \mathbf{s}^{\mathbf{t}}, p^{t}, \mathbf{N}^{t}\right) & =-c q^{t}+p^{t} E\left[\Phi^{t}\right]+\sum_{k=1}^{t-1}\left(s^{k}+m-c\right) E\left[N^{t k}\right] \quad \forall t \leq T \\
\Phi^{t} & =\min \left\{q^{t}, D^{t}\right\} \quad \forall t \leq T \\
N^{t+1, t} & =\Delta^{t} \cdot \Phi^{t} \quad \forall t \leq T \\
N^{t+1, k} & =B^{t k} \cdot N^{t k} \quad \forall k<t \leq T \\
g^{T+1}\left(\mathbf{N}^{T+1}\right) & =\tilde{v}+v \cdot \sum_{k=1}^{T} E\left[N^{T+1, k}\right] \\
s^{t}, q^{t}, p^{t} & \geq 0 \quad \forall t \leq T .
\end{aligned}
$$

In the above formulation, the dependencies of the the random variables on $q^{t}, s^{t}$ and $p^{t}$ are suppressed in the notation. 


\subsection{Reformulation of the DP: One-Stage Representation}

The formulation (1) presented in Section 2.2 is a multi-period dynamic programming formulation, and the expected profit function $g^{t}$ in period $t$ depends not only on the decisions made in that periods, but also on decisions made during all previous periods. In this section, we present an alternative representation of the dynamic program such that it is equivalent to a series of independent single-stage problems.

A key observation that enables the reformulation is that the single-period expected profit function $g^{t}$ can be expressed as a sum of $t$ expressions, where the $k^{\prime}$ th expression depends only on the decisions made in period $k$, for $k \leq t$. For $k<t$, note from the recursive relation,

$$
N^{t k}=B^{t-1, k}\left(s^{k}\right) \cdots B^{k+1, k}\left(s^{k}\right) \cdot \Delta^{k}\left(s^{k}, p^{k}\right) \cdot \min \left\{q^{k}, D^{k}\left(p^{k}\right)\right\}
$$

which depends on $\left(q^{k}, s^{k}, p^{k}\right)$ and not on other decision variables. It follows that the expression of $g^{t}$ in (1) is separable in $\left(q^{k}, s^{k}, p^{k}\right)$ for $k=1, \ldots t$.

For each $t=1,2, \ldots, T$, define $\phi^{t}\left(q^{t}, p^{t}\right)=E\left[\min \left\{q^{t}, D^{t}\left(p^{t}\right)\right\}\right]$. Also, define

$$
\tilde{p}^{t, T}=p^{t}+\delta^{t}\left(s^{t}, p^{t}\right)\left[\left(s^{t}+m-c\right) \sum_{j=t+1}^{T} \alpha^{j-t} \beta^{t}\left(s^{t}\right)^{j-t-1}+v \cdot \alpha^{T-t+1} \beta^{t}\left(s^{t}\right)^{T-t}\right],
$$

where $\beta^{t}\left(s^{t}\right)=E\left[B^{t+1, t}\left(s^{t}\right)\right]$ is the retention rate, and $\delta^{t}\left(s^{t}, p^{t}\right)=E\left[\Delta^{t}\left(s^{t}, p^{t}\right)\right]$ is the conversion rate. It can be shown that the $\tilde{p}^{t, T}$ value above represents the lifetime value of a customer who purchases a single copy at the newsstand. See Kotler and Armstrong (2007) for more information on customer lifetime value (CLV). In the special case of $T=\infty$, the above expression becomes

$$
\tilde{p}^{t, \infty}=p^{t}+\delta^{t}\left(s^{t}, p^{t}\right)\left[\frac{\left(s^{t}+m-c\right) \cdot \alpha}{1-\alpha \cdot \beta^{t}\left(s^{t}\right)}\right] .
$$

Now, define

$$
h^{t, T}\left(q^{t}, s^{t}, p^{t}\right)=\tilde{p}^{t, T} \cdot \phi^{t}\left(q^{t}, p^{t}\right)-c \cdot q^{t}
$$

Proposition 1 below shows that solving the multi-period DP (1) is equivalent to maximizing a series of $h^{t}$ functions separately.

Proposition 1 The dynamic programming formulation (1) is the equivalent to

$$
\max \sum_{t=1}^{T} \alpha^{t} \cdot h^{t, T}\left(q^{t}, s^{t}, p^{t}\right)
$$

If $\left(q^{t}, s^{t}, p^{t}\right)$ maximizes the $h^{t, T}$ function for each $t \leq T$, then $\left\{\left(q^{t}, s^{t}, p^{t}\right) \mid 1 \leq t \leq T\right\}$ is an optimal solution to (1). 
The key idea behind the proof of Proposition 1 is that the lifetime of a single-copy at the newsstand is not affected by any decision that the firm makes in the future periods. Proofs of all results, including Proposition 1, can be found in the appendix.

In this paper, we assume that there is no subscriber at the beginning of the planning horizon, i.e., $N^{1}=0$. In the case of $N^{1}>0$, the objective function of (5) differs only by an additive constant. Thus, Proposition 1 remains valid.

One can interpret the definition of $h^{t, T}$ in (4) as a difference of revenue and cost. In the first term, $\tilde{p}^{t, T}$ represents the expected revenue generated by a single customer who purchases the magazine at the newsstand in period $t$. This revenue consists of (i) the immediate revenue at the newsstand and (ii) the expected subscription and advertising revenue for the remainder of planning horizon. The second term represents the cost of production for the newsstand stocking quantity in period $t$.

The problem of minimizing the single-stage function $h^{t, T}$ is related to a generalization of the classical newsvendor problem, and the details are discussed further in the following sections.

\section{Monopoly Results}

In Section 2, we described the model in a monopolistic setting, which we analyze in this section. (The duopolistic setting will be discussed later in Section 4.) In each of the three subsections, we study the models described in Section 2.2.

\subsection{Monopolist with Quantity Decision}

In this section, we consider Model Q defined in Section 2.2. In this model, both the newsstand price $p^{t}$ and the subscription price $s^{t}$ are exogenously given, and the firm decides on the newsstand stocking quantity $q^{t}$.

Recall from Proposition 1 that the firm's total profit is a separable function of $q^{t}$ 's, namely the sum of $h^{t, T}$ functions. Each $h^{t, T}$ function, consisting of the revenue and cost generated by decision $q^{t}$ over the planning horizon, is independent of all other $q^{t^{\prime}}$ 's, where $t^{\prime} \neq t$. Thus to find the optimal

stocking quantity decision $q^{t}$, we need to consider $h^{t, T}$ only. Each of these separate problems can be reduced to a classical newsvendor problem, where the cost parameter depends on the period index $t$. Proposition 2 below shows how to find the optimal stocking quantity $q^{t}$.

Proposition 2 Consider Model $Q$ in the monopolistic setting. The optimal stocking quantity in 
the $t^{\text {th }}$ period is the newsvendor quantity $q^{t}$ satisfying:

$$
P\left[D^{t}>q^{t}\right]=c / \tilde{p}^{t, T}
$$

where $\tilde{p}^{t, T}$ is the virtual price defined in (2) and (3).

The above result holds both the finite horizon case and the infinite horizon case. We now study the sensitivity of the optimal quantity decision with respect to prices. If the conversion rate $\delta^{t}$ and the retention rate $\beta^{t}$ are not affected by the changes in the subscription price, then the following monotonicity results hold.

Lemma 1 Suppose that the newsstand price is constant, i.e., $p^{t}=p$ for all $t$, and the subscription prices are decreasing (increasing). If the conversion rate $\delta$ and retention rate $\beta$ are independent of subscription prices $s^{t}$ then,

(i) In the infinite horizon case, the optimal stocking quantities $q^{t}$ is decreasing (increasing) in $t$.

(ii) In the finite horizon case, the optimal stocking quantities $q^{t}$ is decreasing (increasing) in $t$ provided

$$
s^{t}+m-c+\alpha \cdot v \cdot \beta \geq v .
$$

The condition (7) in Lemma 1 is a weak requirement and displays an interesting interpretation. Suppose that a firm continues operation for one more period and then salvages the business. Then, a current subscriber generates the profit of $s^{t}+m-c$ in the current period, and this subscriber will continue subscription with probability $\beta$, in which case he is "sold" at the rate of $v$. Thus, the left side of (7) represents the expected total discounted profit of a current subscriber in these two periods. The right side of (7) represents the value of a current subscriber if the firm is salvaged in the current period. Thus, condition (7) means that it is better to run the business for one more period than to salvage it.

It is interesting to note that this condition is not required for the infinite horizon case. However, consider a variation of the model where the manager may salvage the firm and receive $v$ per subscriber (without any fixed salvage value of $\tilde{v}$ ). In such case, it can be shown that the above condition is a sufficient condition for the firm to continue operation and never exercise the salvage option.

It can be seen from the proof of Lemma 1 that if both the newsstand and subscription prices are fixed over time, then the the optimal stocking quantity $q^{t}$ is strictly decreasing in $t$ (in a finite horizon model). We will further explore this effect with numerical examples in Section 5. 


\subsection{Monopolist with Quantity and Subscription Price Decision}

In this section, we add the subscription price to the decision variables of the firm and analyze Model QS of section 2.2. We assume that in each period, the firm chooses a subscription price $s^{t}$ to offer to new subscribers, while the subscription price for the previous subscribers remains unchanged.

Recall that $\delta^{t}(\cdot)$ and $\beta^{t}(\cdot)$ are conversion and retention rates. Typically $\delta^{t}(\cdot)$ and $\beta^{t}(\cdot)$ are decreasing functions of the subscription price $s^{t}$ over an interval $\left[s_{l}^{t}, s_{u}^{t}\right]$. For practical purposes one can assume $s_{l}^{t}=0$ and $s_{u}^{t}=p$. These monotonicity assumptions follow from the real life observation that with the increase in the subscription price more people tend to cancel their subscription and less people tend to subscribe. Moreover, we assume that these rates satisfy the following regularity conditions.

Regularity Conditions (RC): We assume that the following assumptions hold for $\delta^{t}\left(s^{t}\right)$ and $\beta^{t}\left(s^{t}\right): \delta^{t}\left(s^{t}\right)$ and $\beta^{t}\left(s^{t}\right)$ are decreasing in $s^{t} \in \mathbb{R}^{+}$, both are differentiable and the following limits hold:

$$
\begin{aligned}
& 1 \lim _{s^{t} \rightarrow \infty} s^{t} \cdot \delta^{t}\left(s^{t}\right)=0 \\
& 2 \lim _{s^{t} \rightarrow \infty} \beta^{t}\left(s^{t}\right)=0 .
\end{aligned}
$$

The first limit means that the revenue generated through subscription will be 0 when the subscription price is arbitrarily large. Note that this also implies the conversion rate will be 0 . The second limit means that the retention rate will be 0 when the subscription price is arbitrarily large. Hence no one will subscribe and all current subscribes will cancel at high subscription prices. Using these assumptions, we derive the optimal subscription price for the firm.

Proposition 3 Under $(R C)$ in the infinite horizon case, the optimal value for the subscription price $s^{t}$ is either a positive solution to

$$
\frac{\delta^{t}\left(s^{t}, p^{t}\right)}{s^{t}+m-c}+\frac{\alpha \delta^{t}\left(s^{t}, p^{t}\right)}{1-\alpha \cdot \beta^{t}\left(s^{t}\right)} \cdot \frac{\partial \beta^{t}\left(s^{t}\right)}{\partial s^{t}}+\frac{\partial \delta^{t}\left(s^{t}, p^{t}\right)}{\partial s^{t}}=0
$$

or $s^{t}=0$.

Note: A similar result can be obtained for the finite horizon case which is not stated here.

Observation 1: If the revenue from advertisement $(m)$ is 0 , the optimal subscription price $\left(s^{t}\right)$ will be positive, but if the advertisement revenue is a positive value, the optimal subscription price can be 0 .

If the advertisement revenue is equal to 0 , setting subscription price to 0 will yield a high number of subscribers but it will not generate any revenue for the firm. In this setting, increasing 
the subscription price by a small amount $\epsilon>0$ will decrease the number of subscribers, but at the same time will generate a positive subscription revenue. It also will not affect the stand sales. Thus the optimal subscription price will be non-zero when advertisement revenue is 0 .

The second part of the above claim occurs when the revenue generated through advertisement is high enough to offset the decrease in revenue caused by setting a zero subscription price. In other words, the high advertisement revenue justifies lowering the subscription price to zero to attract a larger number of subscribers.

Observation 2: Equation (8) is independent of the stocking quantity $q^{t}$, so the firm will first determine its optimal subscription price and then the optimal stocking quantity calculations will be carried out as in section 3.1. This happens since the subscription revenue increases linearly per customer. Basically, the problem of choosing the optimal subscription price reduces to problem of maximizing revenue per customer, and thus the number of customers does not have any effect. Proposition 4 formalizes this claim.

Proposition 4 The stocking quantity $\left(q^{t}\right)$ decision of a monopolist is determined through equation (6), and in the infinite horizon case the subscription price $\left(s^{t}\right)$ is determined independently of the stocking quantity through equation (8).

Again a similar result can be derived for the finite horizon case.

\subsection{Monopolist with Stocking Quantity, Subscription Price and Newsstand Price Decisions}

In this section we consider Model QSP of section 2.2, where stocking quantity $q^{t}$, subscription price $s^{t}$ and newsstand stand price $p^{t}$ are the decision variables of the firm. In this setting, we consider specific functional forms for subscription and retention rates that make the problem analytically tractable through the one-stage stage representation in section 2.3.

\subsubsection{Optimal Subscription Price}

We assume that the dependency of subscription rate $(\delta)$ on newsstand and subscription prices is of a multiplicative form, i.e. $\delta^{t}\left(s^{t}, p^{t}\right)=\delta^{t_{1}}\left(s^{t}\right) \delta^{t_{2}}\left(p^{t}\right)$. Under this assumption, the optimal value for the subscription price in period $t$ is derived in the same fashion as section 3.2. This follows from the separability of $\delta^{t}(\cdot)$ function in newsstand price $\left(p^{t}\right)$ and subscription price $\left(s^{t}\right)$. The equation determining the optimal subscription price will be independent of the newsstand stocking quantity and the newsstand price, and thus it can be solved separately. 
Proposition 5 In the infinite horizon case of a monopolist with quantity, subscription price and newsstand price decisions, if $\delta^{t}(\cdot)$ and $\beta^{t}(\cdot)$ functions satisfy regularity conditions and $\delta^{t}\left(s^{i}, p^{i}\right)=$ $\delta^{t_{1}}\left(s^{i}\right) \delta^{t_{2}}\left(p^{i}\right)$, the optimal value for the subscription price satisfies equation (8).

\subsubsection{Optimal Stocking Quantity and Newsstand Price}

After we find the optimal subscription price $\left(s^{t}\right)$, the one-stage problem can be reduced to a pricesetting newsvendor problem.

Recall that the newsstand demand $D^{t}(\cdot)$ is a function of the newsstand price $\left(p^{t}\right)$. We need to clarify the type of randomness in the demand function. Two common models used in literature to specify randomness in demand functions are additive and multiplicative forms. In the additive case, we assume that demand is of the form $D^{t}\left(p^{t}\right)=a-b p^{t}+\epsilon$ where $\epsilon$ is a random variable and $a$ and $b$ are strictly positive numbers. We retain the assumption of the multiplicative form of $\delta^{t}(\cdot)$ function and furthermore assume $\delta^{t_{1}}\left(s^{t}\right)$ and $\delta^{t_{2}}\left(p^{t}\right)$ are linear functions. We also assume that the retention rate function $\beta^{t}(\cdot)$ is a linear, decreasing function of subscription price $s^{t}$. In summary, we make the following assumptions with the following notation:

$$
\begin{aligned}
\delta^{t}\left(s^{t}, p^{t}\right) & =\left(a_{s}-b_{s} s^{t}\right)\left(a_{p}+b_{p} p^{t}\right) \\
\beta^{t}\left(s^{t}\right) & =a_{\beta}-b_{\beta} s^{t} \\
\tilde{p}^{t, \infty} & =p^{t}+\frac{\left(a_{s}-b_{s} s^{t}\right)\left(a_{p}+b_{p} p^{t}\right)\left(s^{t}+m-c\right)}{1-\alpha\left(a_{\beta}-b_{\beta} s^{t}\right)} .
\end{aligned}
$$

Notice that there is a one-to-one linear relation between newsstand price $\left(p^{t}\right)$ and virtual price $\left(\tilde{p}^{t, \infty}\right)$. Thus, using the above equations, one can derive:

$$
p^{t}=b_{\tilde{p}^{t, \infty}} \tilde{p}^{t, \infty}+a_{\tilde{p}^{t, \infty}}
$$

where

$$
\begin{aligned}
& b_{\tilde{p}^{t, \infty}}=\left(1+\frac{\left(a_{s}-b_{s} s^{t}\right)\left(s^{t}+m-c\right)}{1-\alpha\left(a_{\beta}-b_{\beta} s^{t}\right)} b_{p}\right)^{-1} \\
& a_{\tilde{p}^{t, \infty}}=-\frac{\left(a_{s}-b_{s} s^{t}\right)\left(s^{t}+m-c\right)}{1-\alpha\left(a_{\beta}-b_{\beta} s^{t}\right)} a_{p} b_{\tilde{p}^{t, \infty}} .
\end{aligned}
$$

Observe that $a_{\tilde{p}^{t, \infty}}$ and $b_{\tilde{p}^{t, \infty}}$ are constants. The above transformations are derived for the infinite horizon case. These transforms can be derived for the finite horizon case in the same fashion. Under the above assumptions, the demand function will be a linear function of virtual price $\tilde{p}^{t}$. 
This directly follows from the linear relation between $p^{t}$ and $\tilde{p}^{t, \infty}$.

$$
\begin{aligned}
D^{t}\left(\tilde{p}^{t, \infty}\right) & =\tilde{a}^{t}-\tilde{b}^{t} \tilde{p}^{t, \infty}+\epsilon \\
\tilde{b}^{t} & =b \cdot b_{\tilde{p}^{t, \infty}} \\
\tilde{a}^{t} & =a-b a_{\tilde{p}^{t, \infty}}
\end{aligned}
$$

In the multiplicative form case the demand function is of the form $D^{t}\left(p^{t}\right)=a\left(p^{t}\right)^{-b} \epsilon$ where $a$ is positive and $b$ is greater than 1 . In this case, after applying the above the transformations, demand as a function of $\tilde{p}^{t, \infty}$ will be $D^{t}\left(\tilde{p}^{t, \infty}\right)=a\left(b_{\tilde{p}^{t, \infty}} \tilde{p}^{t, \infty}+a_{\tilde{p}^{t, \infty}}\right)^{-b} \epsilon$.

The following propositions characterize the optimal newsstand price and newsstands stocking quantity of the firm under the above assumptions. In the rest of this section, we present the results in the infinite horizon case. For the sake of brevity, we drop the $\infty$ from the superscripts.

Proposition 6 If $\delta^{t}(\cdot)=\left(a_{s}-b_{s} s^{t}\right)\left(a_{p}+b_{p} p^{t}\right)$ the optimal $\left(\tilde{p}^{t *}, q^{t *}\right)$ decision of the monopolist facing additive or multiplicative demand functions with a uniform $[0,1]$ noise term, $(\epsilon=U[0,1])$ satisfies the following equations.

- Additive Noise:

$$
\begin{aligned}
q^{t *} & =\tilde{a}^{t}+1-\frac{c}{\tilde{p}^{t *}}-\tilde{b}^{t} \tilde{p}^{t *} \\
0 & =\frac{1}{2}+\tilde{a}^{t}-2 \tilde{b}^{t} \tilde{p}^{t *}-\frac{c^{2}}{2 \tilde{p}^{t * 2}}+c \tilde{b}^{t}
\end{aligned}
$$

- Multiplicative Noise

$$
\begin{aligned}
q^{t *} & =\frac{a\left(\tilde{p}^{t *}-c\right)}{\tilde{p}^{t *}\left(b_{\tilde{p}^{t}} \tilde{p}^{t *}+a_{\tilde{p}^{t}}\right)^{b}} \\
0 & =1-\frac{\tilde{p}^{t *}-c}{2 \tilde{p}^{t *}}-\frac{b b_{\tilde{p}^{t}}\left(\tilde{p}^{t *}-c\right)}{2\left(b_{\tilde{p}^{t}} \tilde{p}^{t *}+a_{\tilde{p}^{t}}\right)}
\end{aligned}
$$

Call the set of $\left(\tilde{p}^{t *}, q^{t *}\right)$ that satisfy the necessary conditions of Proposition $6 \Gamma_{\times}^{t *}$ and $\Gamma_{+}^{t}$ for multiplicative and additive cases respectively. Also define $\Gamma_{+}^{t *}$ as follows:

$$
\Gamma_{+}^{t *}:=\left\{\left(\tilde{p}^{t}, q^{t}\right) \mid\left(\tilde{p}^{t}, q^{t}\right) \in \Gamma_{+}^{t}, b_{\tilde{p}^{t}} \tilde{p}^{t}+a_{\tilde{p}^{t}} \geq 0\right\} \bigcup\left\{\left(-a_{\tilde{p}^{t}} / b_{\tilde{p}^{t}}, \tilde{a}^{t}+1+c b_{\tilde{p}^{t}} / a_{\tilde{p}^{t}}+b a_{\tilde{p}^{t}}\right)\right\}
$$

The following proposition shows how to deduce the optimal stand price $p^{t}$ from the virtual optimal price $\tilde{p}^{t}$. 
Proposition 7 The optimal newsstand price and stocking quantity decision vector $\left(p^{t *}, q^{t *}\right)$ is equal to $\left(b_{\tilde{p}^{t}} \tilde{p}^{t *}+a_{\tilde{p}^{t}}, q^{t *}\right)$, where $\left(\tilde{p}^{t *}, q^{t *}\right)$ is derived as follows:

$$
\left(\tilde{p}^{t *}, q^{t *}\right)=\underset{\left(\tilde{p}^{t}, q^{t}\right) \in \Gamma_{+}^{*} \text { or } \Gamma_{\times}^{*}}{\arg \max } \Pi\left(\tilde{p}^{t}, q^{t}\right)
$$

where $\Pi\left(\tilde{p}^{t}, q^{t}\right)=\tilde{p}^{t} \phi^{t}\left(\tilde{p}^{t}, q^{t}\right)-c q^{t}$ is the newsvendor objective function of the one-stage representation.

\section{Duopoly Results}

\subsection{Formulation}

In this section, we consider a duopolistic environment. Following the previous notation, we introduce the following additions to the model. All the parameters defined before are firm specific, so they will be indexed by subscript $i \in\{1,2\}$ to show that they belong to a specific firm. We also modify the demand functions to show how the competition occurs. The new demand function $D_{i}^{t}$ is defined as follows:

$$
D_{i}^{t}=\Lambda_{i}^{t}+\gamma_{i}\left(\Lambda_{j}^{t}-q_{j}^{t}\right)^{+}
$$

The interpretation of the new demand function is as follows. Each firm $i$ has a separate loyal customer base at time $t$ which is a random number $\Lambda_{i}^{t}$. The firm gets this demand regardless of its competitor's effort. In addition to this demand, the firm also gets a fraction $\gamma_{i}$ of the unsatisfied demand of the competitor $\left(\Lambda_{j}^{t}-q_{j}^{t}\right)^{+}$. Notice that now the effective demand $D_{i}^{t}$ is a function of the vector $\left(p_{i}^{t}, q_{j}^{t}\right)$ and indirectly $p_{j}^{t}$, as each firm's initial demand is a function of its own price $\left(\Lambda_{i}^{t}=\Lambda_{i}^{t}\left(p_{i}^{t}\right)\right)$.

Remark: One can interpret $\gamma_{i}$ as a measure of market separability. If the two markets are very separate (i.e. there is not much overflow of demand), then the $\gamma_{i}$ value is low. If the markets are not separate and customers shift companies easily, this translates into a high value for $\gamma_{i}$. This interpretation is more tangible when the $\gamma_{1}=\gamma_{2}$.

Given the above notation the dynamic programming problem of each firm can be written as follows: Maximize $V_{i}^{1}\left(\mathbf{s}_{i}^{0}, \mathbf{s}_{-i}^{0}, \mathbf{N}_{i}^{1}\right)$, where

$$
\begin{aligned}
V_{i}^{t}\left(\mathbf{s}_{i}^{t-1}, \mathbf{s}_{-i}^{t-1}, \mathbf{N}_{i}^{t}\right) & =\max \quad W_{i}^{t}\left(q_{i}^{t}, q_{-i}^{t}, \mathbf{s}_{i}^{t-1}, \mathbf{s}_{-i}^{t-1}, p_{i}^{t}, p_{-i}^{t}, \mathbf{N}_{i}^{t}\right) \quad \text { for } t \leq T \\
W_{i}^{t}(.) & =g_{i}^{t}\left(q_{i}^{t}, q_{-i}^{t}, \mathbf{s}_{i}^{t}, \mathbf{s}_{-i}^{t}, p_{i}^{t}, p_{-i}^{t}, \mathbf{N}_{i}^{t}\right)+\alpha \cdot V_{i}^{t+1}\left(\mathbf{s}_{i}^{t}, \mathbf{s}_{-i}^{t}, \mathbf{N}_{i}^{t}\right) \quad \text { for } t \leq T \\
V_{i}^{T+1}\left(\mathbf{s}^{T}, \mathbf{N}^{T+1}\right) & =\alpha^{T+1} \cdot g_{i}^{T+1}\left(\mathbf{N}_{i}^{T+1}\right) .
\end{aligned}
$$


Above, the maximization is taken over $\left\{q_{i}^{t}\right\},\left\{q_{i}^{t}, s_{i}^{t}\right\}$, or $\left\{q_{i}^{t}, s_{i}^{t}, p_{i}^{t}\right\}$ depending on the Model Q, QS or QSP. Furthermore, the single-period cost function $g_{i}^{t}$ and the evolution of the quantities of interest are defined as follows:

$$
\begin{aligned}
g_{i}^{t}(.) & =-c_{i} q_{i}^{t}+p_{i}^{t} E\left[\Phi_{i}^{t}\right]+\sum_{k=1}^{t-1}\left(s_{i}^{k}+m_{i}-c_{i}\right) E\left[N_{i}^{t k}\right] \quad \forall t \leq T \\
\Phi_{i}^{t} & =\min \left\{q_{i}^{t}, D_{i}^{t}\right\} \quad \forall t \leq T \\
N_{i}^{t+1, t} & =\Delta_{i}^{t} \cdot \Phi_{i}^{t} \quad \forall t \leq T \\
N_{i}^{t+1, k} & =B_{i}^{t k} \cdot N_{i}^{t k} \quad \forall k<t \leq T \\
g_{i}^{T+1}\left(\mathbf{N}_{i}^{T+1}\right) & =\tilde{v}_{i}+v_{i} \cdot \sum_{k=1}^{T} E\left[N_{i}^{T+1, k}\right] \\
s_{i}^{t}, q_{i}^{t}, p_{i}^{t} & \geq 0 \quad \forall t \leq T .
\end{aligned}
$$

We note that the duopoly magazine competition model with the above demand function was first studied by Ahn and Olsen (2005). In this paper, we extend their model in the sense that we can handle multiple subscription prices in one period easily. We use linear dynamics for conversion and retention rates, which simplifies the model and hence allows us to find analytical solutions. Finally, our approach to the model is different in the sense that we first build a monopoly model with minimal decision variables and then extend it to a competition model with a full array of decision variables. This approach allows for an evaluation of the effect of each decision variable separately and gives insight on how different decision variables affect the total profit. We also present a singlestage reduction of the problem, which links this problem directly to a newsvendor problem with a redefined price/cost structure. In this fashion, we solve the problem of the newsvendor and deduce the optimal solution to the dynamic programming problem from the newsvendor solution.

\subsection{Competition with Quantity Decision}

\subsubsection{Characterization of Equilibria}

In section 4.2.1, we assume there are two firms which will be competing over the quantity they send to the stands, while the subscription and stand prices are fixed. The problem in this case will be reduced to the case of two competing newsvendors. The following proposition shows that the one-stage representation introduced in Proposition 1 is still valid.

Proposition 8 The problem of the firm $i$ in the duopolistic setting facing dynamic programming problem (11) is equivalent to

$$
\max \sum_{t=1}^{T} \alpha^{t} \cdot h_{i}^{t, T}\left(q_{i}^{t}, q_{-i}^{t}, s_{i}^{t}, s_{-i}^{t}, p_{i}^{t}, p_{-i}^{t}\right) .
$$


where $h_{i}^{t, T}($.$) is defined as in (4) with the modification presented in subsection 4.1. If \left(q_{i}^{t}, s_{i}^{t}, p_{i}^{t}\right)$ maximizes the $h_{i}^{t, T}$ function for each $t \leq T$, then $\left\{\left(q_{i}^{t}, s_{i}^{t}, p_{i}^{t}\right) \mid 1 \leq t \leq T\right\}$ is an optimal solution to (11).

Using the above proposition, we can reduce the problem to the problem of two competing newsvendors. The following proposition characterizes the equilibrium point of this competition. Before proceeding to the result, we define the following parameter. Let

$$
\sigma_{i}=c_{i} / \tilde{p}_{i}
$$

This parameter is the ratio of the cost to the virtual price $\tilde{p}$. Recall that virtual price is the expected revenue a single copy sale on stand will generate over the the planning horizon (taking into account the subscription and advertisement revenues).

Next, we assume that the demand is uniformly distributed. Without loss of generality, we assume the demand is $U[0,1]$, and we carry out this assumption in the remainder of section 4.2.

Proposition 9 If the two firms are working in markets which are separate enough (with regard to the overflow parameter $\left.\gamma_{i}\right)$, the equilibrium point $\left(q^{1 *}, q^{2 *}\right)$ of the duopolistic competition of two firms with demand functions $D_{i}=\Lambda_{i}+\gamma_{i}\left(\Lambda_{j}-q_{j}\right)^{+}$where $\Lambda_{i}$ and $\Lambda_{j}$ are $U[0,1]$ is:

1. If $\sigma_{2} \leq \sqrt{2 \sigma_{1} / \gamma_{1}}$ and $\sigma_{1} \leq \sqrt{2 \sigma_{2} / \gamma_{2}}(\dagger)$ the equilibrium point is the unique solution of the following equations in which $q_{1}^{*}, q_{2}^{*} \in[0,1]$ :

$$
\begin{aligned}
& \frac{c_{1}}{\tilde{p_{1}}}=\left(1-q_{1}^{*}\right)+\frac{\gamma_{1}\left(1-q_{2}^{*}\right)^{2}}{2} \\
& \frac{c_{2}}{\tilde{\tilde{p}_{2}}}=\left(1-q_{2}^{*}\right)+\frac{\gamma_{2}\left(1-q_{1}^{*}\right)^{2}}{2} .
\end{aligned}
$$

2. If $\sigma_{2} \geq \sqrt{2 \sigma_{1} / \gamma_{1}}$ and $\sigma_{1} \leq \sqrt{2 \sigma_{2} / \gamma_{2}}(\ddagger)$ the equilibrium point is the unique solution of the following equations in which $q_{1}^{*} \in\left[1,1+\gamma_{1} \sigma_{2}\right]$ and $q_{2}^{*} \in[0,1]$ :

$$
\begin{aligned}
& e_{2} q_{1}^{* 2}+e_{1} q_{1}^{*}+e_{0}=0 \\
& q_{2}^{*}=1-\frac{c_{2}}{\tilde{p}_{2}}
\end{aligned}
$$

where

$$
\begin{aligned}
& e_{2}=\frac{1}{2 \gamma_{1}} \\
& e_{1}=-\left(\frac{1}{\gamma_{1}}+\frac{c_{2}}{\tilde{p}_{2}}\right) \\
& e_{0}=\frac{c_{2}}{\tilde{p}_{2}}-\frac{c_{1}}{\tilde{p}_{1}}+\frac{1}{2} \frac{\gamma_{1} c_{2}^{2}}{\tilde{p}_{2}^{2}}+\frac{1}{2 \gamma_{1}}
\end{aligned}
$$


3. If $\sigma_{2} \leq \sqrt{2 \sigma_{1} / \gamma_{1}}$ and $\sigma_{1} \geq \sqrt{2 \sigma_{2} / \gamma_{2}}$, then analogous result holds as above.

The $\gamma_{i}$ parameters should satisfy $\gamma_{i} \leq\left(1-\sigma_{i}\right) / \sigma_{j}$.

Remark: In many settings, the conditions on $\gamma_{i}$ values are satisfied naturally. In a typical newsvendor problem, where there is an overage (b) and underage (h) cost (this can be an extension to our model that will consider the loss of goodwill if we do not satisfy the demand), we might have $h>b$. It can be shown that $h>b$ is a sufficient condition to guarantee the above mentioned bounds on the $\gamma_{i}$ 's.

\subsubsection{Interpretation of Different Equilibrium Behaviors}

The following argument interprets condition $(\dagger)$. Expand the condition as follows:

$$
\begin{aligned}
& \left.\sigma_{2} \leq \sqrt{2 \sigma_{1} / \gamma_{1}} \Longrightarrow \sigma_{2} \leq \sqrt{2 / \gamma_{1}} \sqrt{\sigma_{1}} \quad \text { (upper bound on } c_{2} / \tilde{p}_{2}\right) \\
& \left.\sigma_{1} \leq \sqrt{2 \sigma_{2} / \gamma_{2}} \Longrightarrow \sigma_{1} \leq \sqrt{2 / \gamma_{2}} \sqrt{\sigma_{2}} \Longrightarrow \sigma_{1}^{2} \gamma_{2} / 2 \leq \sigma_{2} \quad \text { (lower bound on } c_{2} / \tilde{p}_{2}\right)
\end{aligned}
$$

Since $0 \leq \gamma_{i} \leq 1$ and $0 \leq c_{i} \leq \tilde{p}_{i}$ we get that $\sigma_{1}^{2} \gamma_{2} / 2 \leq 1 \leq \sqrt{2 / \gamma_{1}} \sqrt{\sigma_{1}}$ and the above bounds for $\sigma_{2}$ are consistent. Analogous bounds can be derived for $\sigma_{1}$. These bounds show that for both firms to produce an amount in the interval $[0,1]$ (their initial loyal customer demand bounds), their cost structure should not be very different. (For example, if $\sigma_{1}=0.9, \sigma_{2}=0.1$, and $\gamma_{i}=1$, these conditions do not hold.)

Notions of Equilibrium: As stated above, when condition $(\dagger)$ holds, both firms will be producing within the bounds of their initial demand. Another interpretation is that both firms will be using the same functional form of best response functions, i.e. equations (13a) and (13b) which are of the same functional form. We call this type of equilibrium symmetric (Type I). On the other hand, if condition $(\ddagger)$ holds the firms will be using different functional forms of best response functions, i.e. (14a) and (14b). We call this type of equilibrium asymmetric (Type II). Notice in the asymmetric equilibrium one firm is producing more than the upper bound of its initial loyal customer demand.

The following proposition shows that for any two non-identical firms, if the markets are separate enough (thus the overflow coefficient $\gamma_{i}$ 's are low), the symmetric equilibrium emerges.

Lemma 2 For any combination of $\left(c_{1}, p_{1}\right)$ and $\left(c_{2}, p_{2}\right)$, there exists $\gamma^{*}$ such that the equilibrium is symmetric for all $\gamma_{1}, \gamma_{2} \leq \gamma^{*}$. 
The above proposition states that if the firms work in separate enough markets, the symmetric equilibrium will emerge. Another interpretation for $\gamma_{i}$ can be the degree of substitutability of products. Later on we give another interpretation for $\gamma_{i}$ which is useful when firms have different $\gamma_{i}$ values.

We can now interpret condition $(\ddagger)$. As mentioned in the proof, this case is equivalent to firm 1 producing more than the upper bound of its initial demand (more than 1 unit). This will be optimal, if the cost-profit structures of the two firms are very different. For example, firm 2 might be producing much less because of a high $\sigma_{2}$ (cost to price ratio) value relative to firm 1 . In this case, the amount of overflow to firm 1's market justifies firm 1 producing more than 1 unit (the upper bound of its initial demand). Another argument might be that firm 1's $\sigma_{1}$ (cost to revenue ratio) value is so low that it justifies producing a lot in the expectation of firm 2's overflow. Either way, this means that firm 1 is producing more than the upper bound of its initial demand in the anticipation of the overflow of unmet demand from firm 2.

Figure 1 shows how different values of $\sigma_{1}$ and $\sigma_{2}$ correspond to different equilibrium types in an example.

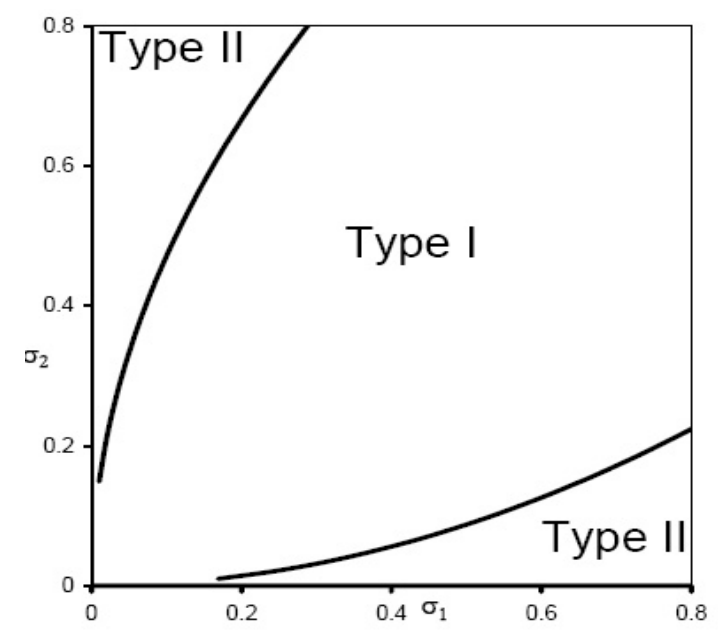

Figure 1: Equilibrium Points

\subsubsection{Convergence of Best Response Functions in Symmetric Equilibrium}

One interpretation of the results in Proposition 9 is as follows. The equations (13a) and (13b) are best response functions to other firm's quantity decision. The following proposition shows that if the firms choose the best response to the other firm's production quantity, they eventually converge 
to the equilibrium point of the competition. Moreover the convergence rate in case 1 of Proposition 9 is linear.

Proposition 10 In the uniform demand and symmetric equilibrium setting, a tâtonnement scheme to solve the system (13a) and (13b) converges linearly to the Nash equilibrium point.

For further information on tâtonnement scheme, please refer to Vives (1990).

\subsection{Competition with Quantity and Subscription Price Decisions}

In this subsection, we consider the case that firms decide both on their optimal quantity and subscription price, while we assume that the newsstand price is exogenously given. In each period, firms choose a subscription price, and coefficients $\delta_{i}$ and $\beta_{i}$ are functions of the subscription price (i.e. $\delta_{i}=\delta_{i}\left(s_{i}^{t}\right)$ and $\left.\beta_{i}=\beta\left(s_{i}^{t}\right)\right)$. The macro and micro level interpretation for $\delta$ and $\beta$ presented in the monopolistic case are still valid. Now we proceed to find the optimal decision of each firm in this setting which leads to the equilibrium.

Proposition 11 If regularity conditions $(\boldsymbol{R} C)$ hold, in the infinite horizon case the optimal value for the subscription price of the firm $i$ competing in a duopoly is either a solution to

$$
\frac{\delta_{i}\left(s_{i}\right)}{s_{i}+m_{i}-c_{i}}+\frac{\alpha \delta_{i}\left(s_{i}\right)}{1-\alpha \cdot \beta_{i}\left(s_{i}\right)} \cdot \frac{\partial \beta_{i}\left(s_{i}\right)}{\partial s_{i}}+\frac{\partial \delta_{i}\left(s_{i}, p_{i}\right)}{\partial s_{i}}=0
$$

or $s_{i}=0$.

As noted by the above proposition, in this case the two firms first decide on the optimal subscription price like in the monopolistic case. This follows from the observation that each firm's optimal subscription price decision is independent of the other firm's quantity and price decision as seen in equation (15). As a result of this independence, each firm will set its subscription price like a monopolist through equation (15), and the problem reduces to the same problem as the previous section.

Proposition 12 Two firms (as in the setting of Proposition 9) competing with quantity and subscription price first set their subscription prices like a monopolist and then choose their production level as in Proposition 9. This decision will determine the unique equilibrium point. 


\section{Numerical Analysis}

In this section, we look into a numerical analysis of the above models and analyze how certain parameters affect the outcome of the model. Our goal here is to focus on certain observed behaviors of the model that are interesting and characterize optimal stand price $\left(p^{*}\right)$, subscription price $\left(s^{*}\right)$, production quantity $\left(q^{*}\right)$, profit $(\Pi)$, subscription rate $(\delta)$ and retention rate $(\beta)$.

\subsection{Effect of Increasing the Current Subscribers' Sensitivity to the Subscription Price in the Monopolistic Setting}

This effect is captured through the change in the $b_{\beta}$ parameter of the retention rate (recall that the retention rate is $\left.\beta(s)=a_{\beta}-b_{\beta} s\right)$. As one might expect, with the increase in the sensitivity of customers, the optimal subscription price decreases, and as a result of this, the subscription rate $(\delta)$ increases (recall that subscription rate: $\delta(s, p)=\delta^{1}(s) \delta^{2}(p)=\left(a_{s}-b_{s} s\right)\left(a_{p}+b_{p} p\right)$ ). The optimal production quantity and the total profit also decrease. An interesting behavior we observe in this case relates to the retention rate the firm faces (see Figure 2 for more details). The retention rate of the firm does not change monotonically with the change in the sensitivity of subscribers. This means for two firms with the same cost structure, the firm with more sensitive subscribers might show a higher retention rate than the other one. This illustrates that as the sensitivity of the customers to subscription price increases, firms do not adjust for this change with the same speed. They prefer to suffer from a lower retention rate but generate profit at a higher price from a smaller customer base. When customers become too sensitive, loss of advertisement revenue forces firms to lower their subscription price to increase the retention rate. This behavior is observed in monopoly models both with and without stand price decisions.

\subsection{Effect of an Increase in Spot-buyers' Sensitivity to the Stand Price}

This effect is captured through the change in the $b_{p}$ parameter of the subscription rate (recall that subscription rate is $\left.\delta(s, p)=\delta^{1}(s) \delta^{2}(p)=\left(a_{s}-b_{s} s\right)\left(a_{p}+b_{p} p\right)\right)$. See Figure 3 for more details. An interesting observation is that with the increase in the sensitivity of spot-buyers, the total profit of the firm increases. Indeed, as stand-price sensitivity increases, more spot-buyers tend to subscribe and pay a lower subscription price, which increases the revenue of the firm through advertisement. Here, we also observe that with the increase in stand-price sensitivity, the stand price that the firm sets increases. This shows that if a firm is facing a customer base which is very sensitive to stand price, it can take advantage of this behavior and execute a reasonable increase in stand price to shift spot-buyers to subscribers, hence gaining more profit. 


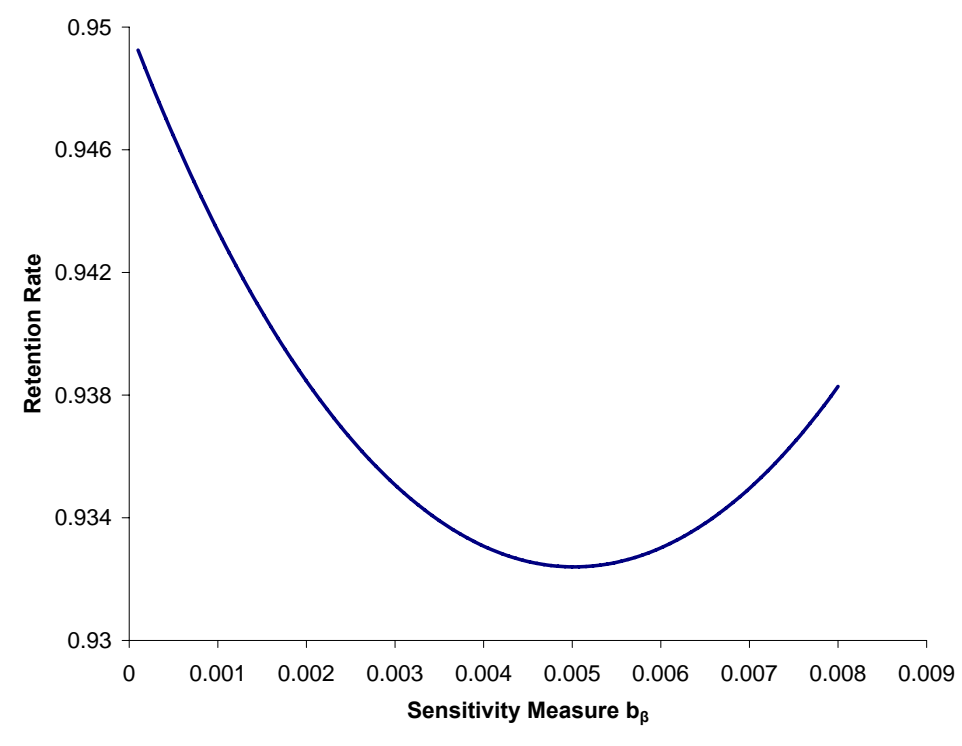

\begin{tabular}{cccccccc}
\hline$m$ & $c$ & $a_{s}$ & $b_{s}$ & $a_{p}$ & $b_{p}$ & $a_{\beta}$ & $\alpha$ \\
\hline 8 & 1 & 0.1 & 0.0045 & 0.3 & 0.001 & 0.05 & 0.95
\end{tabular}

Figure 2: Retention Rate as a Function of Subscribers' Sensitivity to Subscription Price

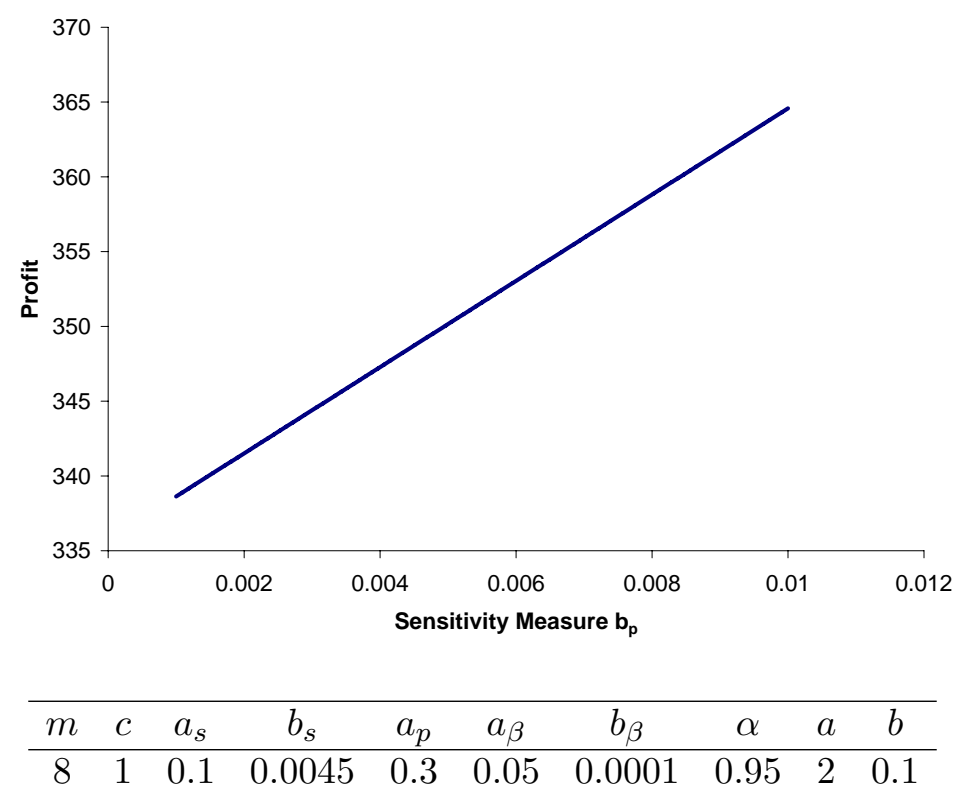

Figure 3: Profit as a function of Sensitivity of Spot-buyers to Stand Price

\subsection{Value of Optimization}

In many real-life settings, companies act as price takers with regard to stand price. In this setting, the two levers that a company has are the subscription price and the quantity it produces. In this 
Table 1: Effect of Optimizing Production Quantity and Subscription Price

\begin{tabular}{|c|c|c|c|c|}
\hline \multirow{3}{*}{ a. Naive Firm } & \multicolumn{3}{|c|}{$O p t(q)-N o O p t$} & \multirow{3}{*}{$\frac{O p t(s, q)-O p t(q)}{O p t(q)}$} \\
\hline & & NoOpt & & \\
\hline & $e_{q}=5 \%$ & $e_{q}=10 \%$ & $e_{q}=15 \%$ & \\
\hline$e_{s}=5$ & $1.5186 \%$ & $6.3637 \%$ & $15.5557 \%$ & $0.0670 \%$ \\
\hline$e_{s}=10$ & $1.5201 \%$ & $6.3710 \%$ & $15.5751 \%$ & $0.2683 \%$ \\
\hline$e_{s}=15$ & $1.5229 \%$ & $6.3832 \%$ & $15.6076 \%$ & $0.6058 \%$ \\
\hline
\end{tabular}

b. Responsive Firm

\begin{tabular}{lcccc} 
& $e_{q}=5 \%$ & $e_{q}=10 \%$ & $e_{q}=15 \%$ & \\
\hline$e_{s}=5$ & $1.5184 \%$ & $6.3635 \%$ & $15.5553 \%$ & $0.0669 \%$ \\
$e_{s}=10$ & $1.5110 \%$ & $6.3704 \%$ & $15.5736 \%$ & $0.2683 \%$ \\
$e_{s}=15$ & $1.5226 \%$ & $6.3820 \%$ & $15.6043 \%$ & $0.6057 \%$ \\
\hline
\end{tabular}

\begin{tabular}{cccccccccccc}
\hline$m$ & $c$ & $a_{s}$ & $b_{s}$ & $a_{p}$ & $b_{p}$ & $a_{\beta}$ & $b_{\beta}$ & $\alpha$ & $a$ & $b$ & $p$ \\
\hline 30 & 27.5 & 0.5 & 0.009 & 0.3 & 0.001 & 0.05 & 0.0001 & 0.95 & 30 & 0.5 & 28
\end{tabular}

section, we try to compare the effect of optimizing these values on the profit of the firm. As our base case, we consider the setting characterized by Table 1 .

In this case, the optimal subscription price and production quantity are $\left(s^{*}, q^{*}\right)=(26.128,16.467)$. We consider two behavior types of firm: naive firms and responsive firms. A naive firm chooses both its production quantity and subscription price randomly around the optimal decisions. A responsive firm chooses its subscription price randomly around the optimal subscription price and then chooses the optimal production quantity for its randomly chosen subscription price.

In order to evaluate the performance of a naive firm, we assume that the firm selects $(\tilde{s}, \tilde{q})$ decision according to $\left(\tilde{s}=s^{*} \pm e_{s} \cdot s^{*}, \tilde{q}=q^{*}\left(s^{*}\right) \pm e_{q} \cdot q^{*}\left(s^{*}\right)\right)$, where both $e_{s}$ and $e_{q}$ take values of $5 \%$, $10 \%$ and $15 \%$. These errors are uniformly distributed, and we implement it using a discretized grid of size $501 \times 501$. In the case of a response firm, the firm selects $\left(\tilde{s}=s^{*} \pm e_{s} \cdot s^{*}, \tilde{q}=q^{*}(\tilde{s}) \pm e_{q} \cdot q^{*}(\tilde{s})\right)$, where $e_{s}$ is defined similarly as above, and $q^{*}(\tilde{s})$ represents the optimal choice of $q$ for given $\tilde{s}$.

In Table 1, NoOpt refers to the average objective function value when the $(s, q)$ vector is chosen randomly in the relevant range. $\operatorname{Opt}(q)$ shows the case when $s$ is chosen randomly and then an optimization problem is solved to find the corresponding optimal $q$. Opt $(s, q)$ refers to the case where both $s$ and $q$ are solved optimally. This example was constructed in a way that two criteria are met, namely $s^{*} \leq p$ and $c \leq p$. From the above example, we observe that the performance of the firm (regardless of being naive or responsive) certainly improves when the firm optimizes both 
$q$ and $s$; however, most of the improvement can be captured by optimizing over a single decision $q$, as evidenced by the last column of the table. This observation is consistent with the range of parameters that we have tested. It is notable that if these conditions are relaxed, specifically if the stand price is chosen to be lower than the production cost, the magnitude of profit improvement from optimization dramatically increases. Furthermore, in such cases we observe that the value from optimizing subscription rate $s$ is higher than that of subsequently optimizing production quantity $q$.

Another interesting observation related to the relationship between demand price and cost can be made. As mentioned previously, in the monopolistic setting with quantity and subscription decisions for the finite horizon case, the optimal quantity is given by the following formula:

$$
1-F_{D}(q)=\frac{c}{p+(s+m-c) \delta \sum_{j=0}^{T-i}(\alpha \beta)^{j}+\alpha^{T-i} v \beta^{T-i} \delta} \cdot
$$

Discarding the second and third terms in the denominator reduces the problem to the case of a newsvendor with no subscription and advertisement revenue. In that case, for the company to be profitable it is required that $c \leq p$, but as the above relation suggests, it is quite possible for a subscription-based firm to price its stand price below the production cost and still make a profit, due to future advertisement and subscription revenues.

It is also notable that if the regularity condition of Lemma $1\left(s^{t}+m-c+\alpha \cdot v \cdot \beta \geq v\right)$ holds, the optimal quantity decision will be increasing in the planning horizon. Ahn and Olsen (2005) observe the same behavior. Since they consider the end-of-horizon values to be 0 , the values in their model automatically satisfy the above regularity condition. Figure 4 shows how the optimal quantity decision changes with the increase in the planning horizon.

\subsection{Effect of Awareness of Existing Competition in the Duopolistic Setting}

In this section, we study the value of considering the competitor's effect on a firm's profit. In a duopolistic setting, two firms can operate on one of the following situations.

1. Both firms are aware of the competition and optimize their decisions considering the other firm's actions.

2. One firm is aware of the competition but the other one is not.

3. Both firms are oblivious of competition. 


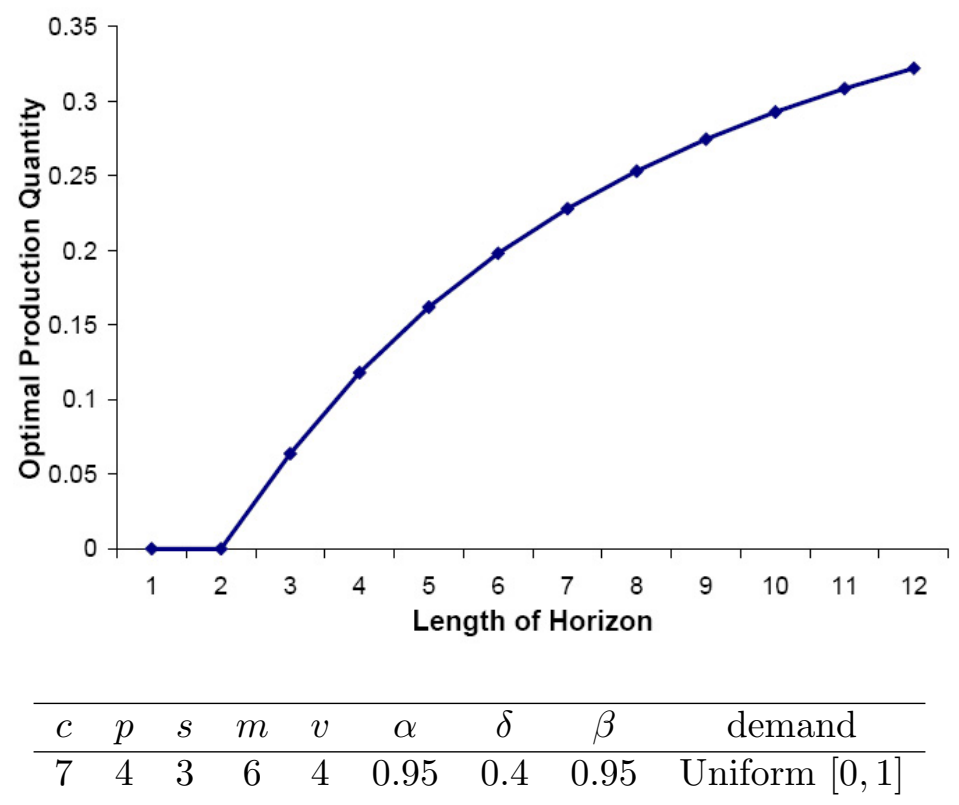

Figure 4: Effect of Planning Horizon's Length on Production Quantity

In the following subsections, we present numerical examples of how certain variables affect the profit of the firm in the settings described above. Below, we suppose that the stand price $p$ is exogenously given and that the decisions include both the quantity $q$ and the subscription price $s$.

\subsubsection{Effect of an Increase in Production Cost for Symmetric Firms}

Figure 5 illustrates the profit improvement of one firm that takes into account the existence of its competitor, while the competitor remains oblivious of competition.

As the cost of production increases (see Figure 5), it becomes more important to consider competition, when a firm decides on the optimal production quantity. The firms are identical in this simulation, and each line shows this effect at a certain level of market separability $\gamma$. For the ease of exposition, $\gamma_{1}$ and $\gamma_{2}$ are taken equal $\left(\gamma_{1}=\gamma_{2}=\gamma\right)$.

\subsubsection{Effect of an Increase in the Stand Price for Symmetric Firms}

Figure 6 shows the value of considering competition as a function of the stand price, which is exogeneously given. Like the previous case, the graph shows this value for various levels of market separability $\gamma$. 


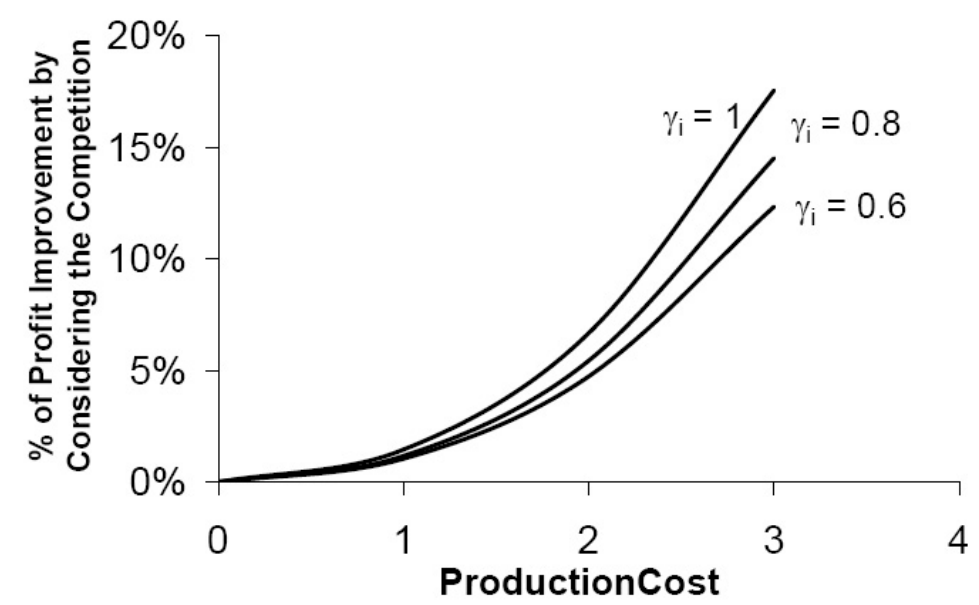

\begin{tabular}{ccccccccc}
\hline$m$ & $a_{s}$ & $b_{s}$ & $a_{p}$ & $b_{p}$ & $a_{\beta}$ & $b_{\beta}$ & $\alpha$ & $p$ \\
\hline 13 & 0.1 & 0.005 & 0.3 & 0.001 & 0.05 & 0.001 & 0.9 & 6 \\
\hline
\end{tabular}

Figure 5: Effect of Cost Increase on Value of Considering the Competition

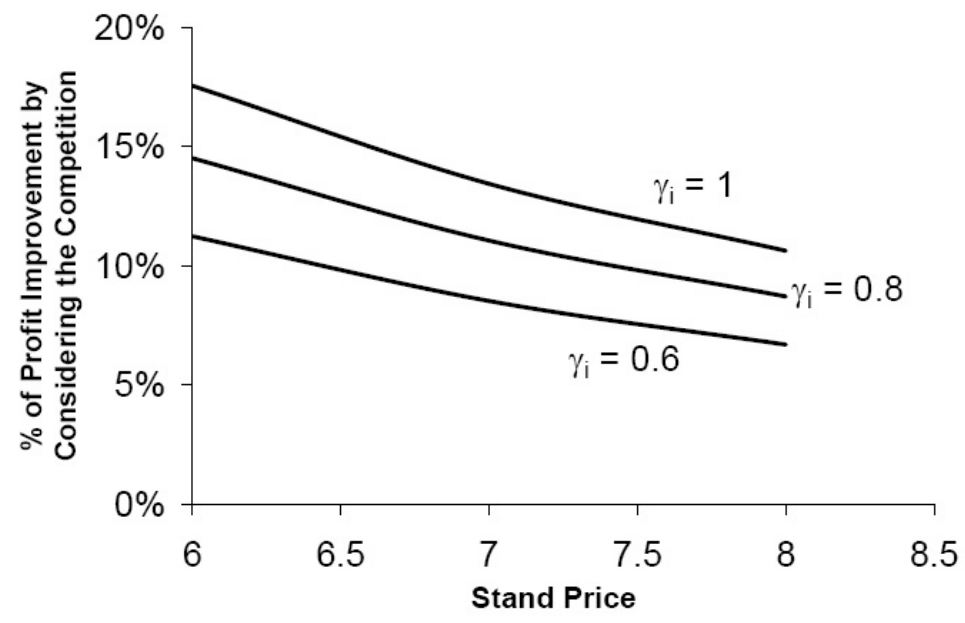

\begin{tabular}{ccccccccc}
\hline$m$ & $a_{s}$ & $b_{s}$ & $a_{p}$ & $b_{p}$ & $a_{\beta}$ & $b_{\beta}$ & $\alpha$ & $c$ \\
\hline 13 & 0.1 & 0.005 & 0.3 & 0.001 & 0.05 & 0.001 & 0.9 & 3 \\
\hline
\end{tabular}

Figure 6: Effect of Stand Price Increase on Importance of Considering the Competition

\subsubsection{Value of Considering Competition for Asymmetric Firms}

In this subsection, we compare the benefits of considering the competition for asymmetric firms. We analyze two types of asymmetry: asymmetry in the cost structure or in the loyalty of customers.

- Cost Structure Asymmetry: 

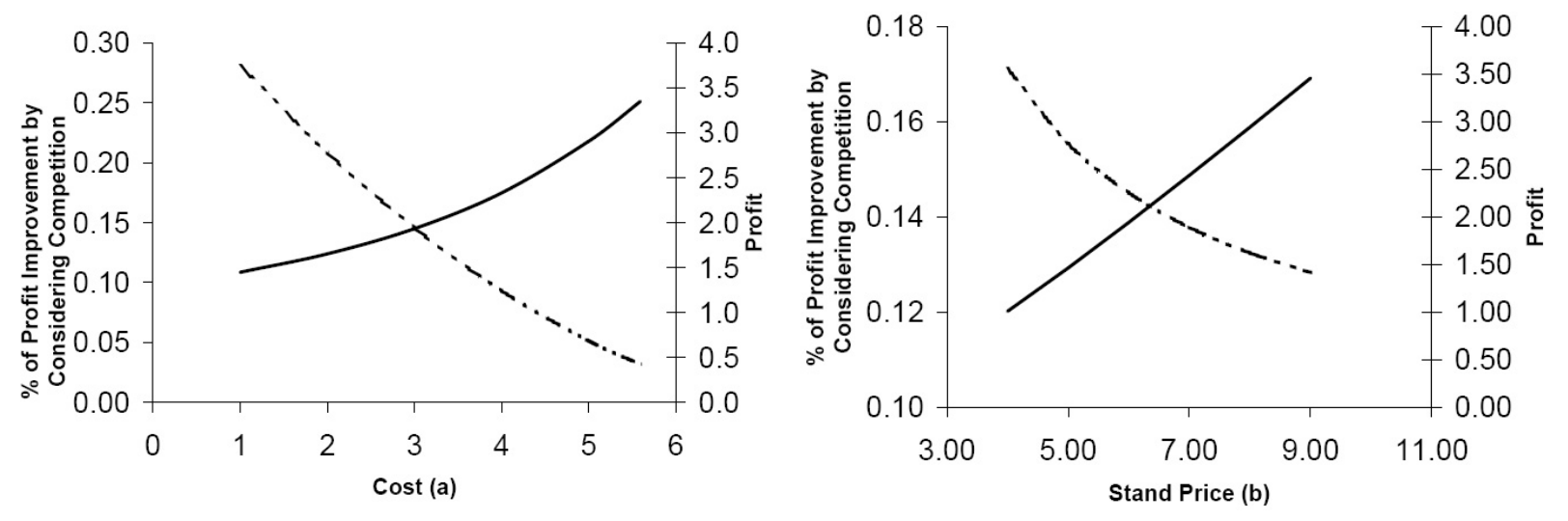

\begin{tabular}{ccccccccc}
\hline$m$ & $a_{s}$ & $b_{s}$ & $a_{p}$ & $b_{p}$ & $a_{\beta}$ & $b_{\beta}$ & $\alpha$ & $\gamma$ \\
\hline 13 & 0.1 & 0.005 & 0.3 & 0.001 & 0.05 & 0.001 & 0.9 & 0.2
\end{tabular}

In the cost graph $c_{1}=3$ and $p=6$ while $c_{2}$ is varying, and in the price graph $c=3$ and $p_{1}=6$ while $p_{2}$ is varying.

The dotted line is the profit, and the solid line shows the percentage of increase in profit by considering competition. The profits are calculated after considering the competition.

Figure 7: Effect of Cost Structure Asymmetry on Competition

Figure 7 shows how the profit of a company changes when the cost of production or the stand price increase. As one expects, profit decreases in the first case and increases in the second case. The interesting behavior can be seen by comparing this trend with the trend of a firm's gain by considering competition. It is more important for high cost-basis companies to consider competition than low cost-basis companies. Figure 7-b shows that companies that enjoy a higher stand price gain less from considering the competition effect than the companies with a lower stand price.

- Customer Loyalty Asymmetry:

In this subsection, we introduce a new interpretation for the $\gamma$ parameter. Previously, we described $\gamma$ as the degree of separation of the markets. This interpretation is more applicable when $\gamma$ values of firms are equal. Another interpretation of $\gamma$ is as loyalty of customers. A firm with a high $\gamma$ value has more loyal customers since its customers shift to the other firm at a lower rate. Alternatively, one can interpret $\gamma$ as the attraction power of one firm. The firm with a higher $\gamma$ can attract the unmet demand of the other firm at a higher rate. Figure 8 shows how firms with different $\gamma$ values benefit from competition.

In this case, we consider two firms which are identical in all parameters and are only different in their $\gamma$ values. The graphs show how one firm's profit from considering competition changes when its $\gamma$ value changes. 

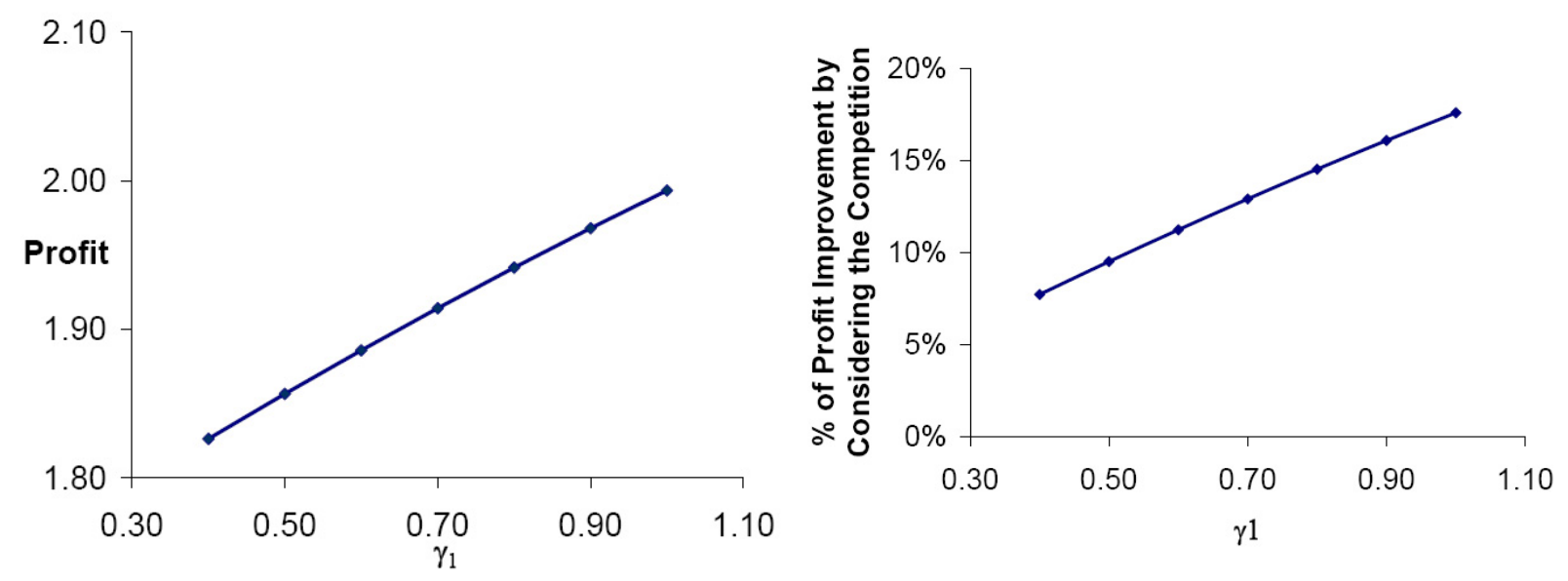

\begin{tabular}{ccccccccccc}
\hline$m$ & $a_{s}$ & $b_{s}$ & $a_{p}$ & $b_{p}$ & $a_{\beta}$ & $b_{\beta}$ & $\alpha$ & $\gamma_{2}$ & $c$ & $p$ \\
\hline 13 & 0.1 & 0.005 & 0.3 & 0.001 & 0.05 & 0.001 & 0.9 & 0.7 & 3 & 6
\end{tabular}

Figure 8: Effect of Customer Loyalty Asymmetry

We observe that as firm 1's customer attraction power $\gamma_{1}$ increases, the profit improvement benefit resulting from considering the effect of competition also increases. This can be explained by the fact that the increased attraction power of a firm results in a higher amount of overflow demand from the competitor, which when properly accounted for results in a higher increase in revenue. In the extreme case where $\gamma$ is equal to zero, the firm does not gain anything by considering the effect of competition.

\section{Conclusions}

In this paper, we formulate the problem of a firm which has two sources of revenue: 1) sales of its product either through direct sales or subscription and 2) advertisement revenue. We propose a model that captures the dynamics of customer subscription, retention and attrition. Specifically, we assume certain functional forms for the rate at which firms acquire new subscribers and the rate at which current subscribers unsubscribe. Based on these dynamics, we formulate the problem of a profit maximizing firm as a dynamic program.

This problem can be hard to solve in the original format, since the revenue generated in each period is affected by all of the decisions taken in any of the previous stages. The revenue of each period depends on the number of customers which subscribed in the previous stages, which is affected by the pricing and production quantity decisions of every stage. We propose a method to reduce this multi-stage problem to a series of single-stage problems by introducing the concept of virtual price. This price represents the revenue generated by a single copy sold over the horizon, 
as opposed to only the revenue it generates in the period it is sold. Using this concept and taking advantage of the functional form of the dynamics of the system, we are able to reduce the problem of the firm to a series of single-stage newsvendor problems. Each of these problems can be easily solved, and the result can be translated to the multi-stage form. This line of analysis is carried over for the monopolistic case in three different scenarios: 1) when a firm has only a production quantity decision, and the subscription and stand prices are exogenous and fixed; 2) when a firm has the production quantity decision and also should decide on the subscription price, but the stand price is fixed; and finally, 3) when the firm has to choose the production quantity and both the subscription and stand prices.

We then consider a duopolistic setting where each firm benefits from the overflow of unsatisfied demand from the other firm. We study different types of equilibria that can emerge in this situation and provide economic interpretations for each type of equilibrium. We use a demand function which can capture the concepts of 1) customer attraction power and 2) customer loyalty/market separability.

Finally, we perform a numerical analysis on the model. We analyze the effect of increasing certain parameters on customer retention and profit of the firm. We also compare the bottom-line impact of each decision variable (i.e. production quantity, subscription price, and stand price) by examining the incremental benefit of optimizing each one. The results show that each of these decisions can be important for the firm and neither of them should be dismissed. We show that as the cost of production and/or the length of the planning horizon increases, it becomes more important for symmetric firms to consider the effect of competition, whereas the increase in the stand price has the opposite effect.

The case of asymmetric firms is also studied to identify which firms benefit more in a competitive setting. We show that firms which have more loyal customers benefit more from the competition. We also consider the impact of production cost and stand price on the value of competition.

For future work, we intend to extend the duopolistic result to a general competitive case where more than two firms compete. We would also like to consider more general functional forms for the dynamics of the model and derive numerical solutions in these cases. We will also try to extend our model to determine the optimal price and user-accessability for online web versions of magazines.

\section{Acknowledgements}

We would like to thank our student Nicklas Volbi and the CJR managing board, including Michael Hoyt and Dennis Giza, for sharing historical data that motivated our models and validated our 
analysis.

\section{References}

Ahn, H., T. L. Olsen. 2005. Inventory competition with subscription. Working Paper .

Bernstein, F., A. Federgruen. 2004. A general equilibrium model for industries with price and service competition. Operations Reseacrh 52 868-886.

Bertrand, J. 1883. Theorie mathematique de la richesse sociale. Journal des Savants 67 499-508.

Bitran, G. R., S. E. V. Mondschein. 1995. Periodic pricing of seasonal products in retailing. Management Science $\mathbf{4 3}$ 64-79.

Chen, X., D. Simchi-Levi. 2004a. Coordinating inventory control and pricing strategies with random demand and fixed ordering cost: The infinite horizon case. Mathematics of Operations Research $29698-723$.

Chen, X., D. Simchi-Levi. 2004b. Coordinating inventory control and pricing strategies with random demand and fixed ordering cost: The finite horizon case. Mathematics of Operations Research $52887-896$.

Cournot, A. 1838 - 1897, English translation by N.T. Bacon. Recherches sur les principes mathmatiques de la thorie des richesses (Researches into the Mathematical Principles of the Theory of Wealth). McMillan (English Translation).

Edgeworth, F. Y. 1888. The mathematical theory of banking. Journal of Royal Statistical Society $51113-127$.

Federgruen, A., A. Heching. 1998. Combined pricing and inventory control under uncertainty. Management Science 44 285-300.

Feng, Y., G. Gallego. 1995. Optimal starting times for end-of-season sales and optimal stopping times for promotional fares. Management Science 41 1371-1391.

Gallego, G., G. van Ryzin. 1994. Optimal dynamic pricing of inventories with stochastic demand over finite horizons. Management Science 40 999-1020.

Kirman, A. P., M. Sobel. 1974. Dynamic oligopoly with inventories. Econometrica 43 279-287. 
Kotler, P., G. Armstrong. 2007. Principles of Marketing. 12th ed. Prentice Hall.

Lippman, S. A., K. F. McCardle. 1997. The competitive newsboy. Operations Research 45 54-65.

Parlar, M. 1988. Game theoretic analysis of substitutable product inventory problem with random demand. Naval Research Logistics 35 397-409.

Petruzzi, N. C., M. Dada. 1999. Pricing and the newsvendor problem: A review with extensions. Operations Research 47 183-194.

Porteus, E. L. 2002. Foundations of Stochastic Inventory Theory. Stanford University Press.

Smith, S., D. Achabal. 1998. Clearance pricing and inventory policies for retail chains. Management Science $44285-300$.

Topkis, D. M. 1979. Equilibrium points in nonzero-sum n-person submodular games. SIAM Journal of Control and Optimization 17 773-787.

Topkis, D. M. 1998. Supermodularity and Complementarity. Princeton University Press.

Vives, X. 1990. Nash equilibrium with strategic complementarities. Journal of Mathematical Economics 19 305-321.

Zipkin, P. H. 2000. Foundations of Inventory Management. McGraw-Hill. Audit Bureau Circulation. http://www.accessabc.com/index.html.

Magazine Publishers of America. http://www.magazine.org.

The Magazine Handbook. http://www.magazine.org/content/Files/MPAHandbook06.pdf 


\section{A. Appendix}

\section{A.1 Proof of Proposition 1}

Let the objective function of the dynamic programming formulation of the $T$ period problem be denoted by $J_{T}$. From (1):

$$
J_{T}=\sum_{t=1}^{T} \alpha^{t}\left[-c q^{t}+p^{t} \phi^{t}+\sum_{k=1}^{t-1}\left(s^{k}+m-c\right) E\left[N^{t k}\right]\right]+\alpha^{T+1}\left[\tilde{v}+v \cdot \sum_{k=1}^{T} E\left[N^{T+1, k}\right]\right] .
$$

From the definition of $N^{t k}$, we have $E\left[N^{t k}\right]=\beta^{t-k} \delta \phi$ for all $k \leq t-1$ and $E\left[N^{t, t-1}\right]=\delta \cdot \phi$. Replacing these values in the objective function and expanding the summations we obtain:

$$
\begin{aligned}
J_{T} & =\alpha\left[-c q^{1}+p^{1} \phi^{1}\right] \\
& +\alpha^{2}\left[-c q^{2}-p^{2} \phi^{2}+\left(s^{1}+m-c\right) \delta^{1} \phi^{1}\right] \\
& +\alpha^{3}\left[-c q^{3}-p^{3} \phi^{3}+\left(s^{1}+m-c\right) \beta^{1} \delta^{1} \phi^{1}+\left(s^{2}+m-c\right) \delta^{1} \phi^{2}\right] \\
& +\cdots \\
& +\alpha^{T+1}\left[\tilde{v}+v \cdot\left(\beta^{1} \delta^{1} \phi^{1}+\left(\beta^{2}\right)^{T-2} \delta^{2} \phi^{2}+\ldots\right)\right]
\end{aligned}
$$

Now collect all the terms with $\left(q^{t}, s^{t}, p^{t}\right)$ decisions together

$$
\begin{aligned}
J_{T} & =\alpha\left[-c q^{1}+p^{1} \phi^{1}+\sum_{k=1}^{T-1}\left(s^{1}+m-c\right)\left(\beta^{1}\right)^{k-1} \delta^{1} \phi^{1} \alpha^{k}+\alpha^{T} v\left(\beta^{1}\right)^{T-1} \delta^{1} \phi^{1}\right] \\
& +\alpha^{2}\left[-c q^{2}-p^{2} \phi^{2}+\sum_{k=2}^{T-1}\left(s^{2}+m-c\right)\left(\beta^{2}\right)^{k-2} \delta^{2} \phi^{2} \alpha^{k-1}+\alpha^{T-1} v\left(\beta^{2}\right)^{T-2} \delta^{2} \phi^{2}\right] \\
& +\alpha^{3}\left[-c q^{3}-p^{3} \phi^{3}+\sum_{k=3}^{T-1}\left(s^{3}+m-c\right)\left(\beta^{3}\right)^{k-3} \delta^{3} \phi^{3} \alpha^{k-2}+\alpha^{T-2} v\left(\beta^{3}\right)^{T-3} \delta^{3} \phi^{3}\right] \\
& +\ldots \\
& +\alpha^{T+1} \tilde{v}
\end{aligned}
$$

Rearranging the above expressions, we get

$$
\begin{aligned}
J_{T} & =\alpha\left\{\left[p^{1}+\delta^{1}\left(\left(s^{1}+m-c\right) \sum_{k=1}^{T-1} \alpha^{k}\left(\beta^{1}\right)^{k-1}+v \cdot \alpha^{T}\left(\beta^{1}\right)^{T-1}\right)\right] \phi^{1}-c q^{1}\right\} \\
& +\alpha^{2}\left\{\left[p^{2}+\delta^{2}\left(\left(s^{2}+m-c\right) \sum_{k=2}^{T-1} \alpha^{k-1}\left(\beta^{2}\right)^{k-2}+v \cdot \alpha^{T-1}\left(\beta^{2}\right)^{T-1}\right)\right] \phi^{2}-c q^{2}\right\} \\
& +\alpha^{3}\left\{\left[p^{3}+\delta^{3}\left(\left(s^{3}+m-c\right) \sum_{k=3}^{T-1} \alpha^{k-2}\left(\beta^{3}\right)^{k-3}+v \cdot \alpha^{T-2}\left(\beta^{3}\right)^{T-1}\right)\right] \phi^{3}-c q^{3}\right\} \\
& +\ldots+\alpha^{T+1} \tilde{v}
\end{aligned}
$$


Finally changing the summation limits we get

$$
\begin{aligned}
J_{T} & =\alpha\left\{\left[p^{1}+\delta^{1}\left(\left(s^{1}+m-c\right) \sum_{k=2}^{T} \alpha^{k-1}\left(\beta^{1}\right)^{k-2}+v \cdot \alpha^{T}\left(\beta^{1}\right)^{T-1}\right)\right] \phi^{1}-c q^{1}\right\} \\
& +\alpha^{2}\left\{\left[p^{2}+\delta^{2}\left(\left(s^{2}+m-c\right) \sum_{k=3}^{T} \alpha^{k-2}\left(\beta^{2}\right)^{k-3}+v \cdot \alpha^{T-1}\left(\beta^{2}\right)^{T-2}\right)\right] \phi^{2}-c q^{2}\right\} \\
& +\alpha^{3}\left\{\left[p^{3}+\delta^{3}\left(\left(s^{3}+m-c\right) \sum_{k=4}^{T} \alpha^{k-3}\left(\beta^{3}\right)^{k-4}+v \cdot \alpha^{T-2}\left(\beta^{3}\right)^{T-3}\right)\right] \phi^{3}-c q^{3}\right\} \\
& +\ldots+\alpha^{T+1} \tilde{v}
\end{aligned}
$$

Thus we have

$$
J_{T}=\sum_{t=1}^{T} \alpha^{t} \cdot h^{t, T}\left(q^{t}, s^{t}, p^{t}\right)+\alpha^{T+1} \tilde{v} \quad \text { where } \quad h^{t, T}\left(q^{t}, s^{t}, p^{t}\right)=\tilde{p}^{t, T} \cdot \phi^{t}\left(q^{t}, p^{t}\right)-c \cdot q^{t}
$$

Notice that $\alpha^{T+1} \tilde{v}$ is constant and has no effect on the optimal choice of decision variables, therefore the $\alpha^{T+1} \tilde{v}$ term can be eliminated from the optimization problem.

\section{A.2 Proof of Proposition 2}

The proof is based on the first order conditions of the one-stage representation of the problem. First, notice that the objective function of the firm is a separable function of quantities produced in each

period (i.e. $\left.h^{t, T}().\right)$. Each of these separate functions is a classical newsvendor problem which has a concave objective function, and thus the first order conditions are necessary and sufficient to find the optimal point.

Note the optimal value of production in the $t^{t h}$ period of a $T$ horizon problem with $q^{* t T}$. This value can be determined using the $J_{T}$ functions, defined in appendix A.1. Taking the derivative of $J_{T}$ with respect to $q^{t}$ and setting it equal to 0 yields equation (6).

Taking the limit of this optimal value yields the same result for the infinite horizon case. The infinite horizon solution can also be derived directly by optimizing the one-stage representation of the infinite horizon problem.

As mentioned above, in both cases the problem is sum of multiple separable classic newsvendor problems which each has a concave objective function, and thus the first order conditions are both necessary and sufficient.

\section{A.3 Proof of Lemma 1}

Since in this lemma the retention rate $\left(\beta^{t}\right)$ does not depend on the subscription price $\left(s^{t}\right)$, the $t$ superscript is dropped for brevity. 
(i) From equation (3) and (6) we know the optimal production quantity value is determined through:

$$
1-F_{D}\left(q^{t}\right)=\frac{c}{p^{t}+\delta^{t}\left(p^{t}\right)\left[\left(s^{t}+m-c\right) \cdot \alpha /\left(1-\alpha \cdot \beta^{t}\right)\right]} .
$$

As we are considering the setting where the conversion rate $(\delta)$ and the retention rate $(\beta)$ are independent of the subscription price $\left(s^{t}\right)$, decreasing the subscription price will increase $1-F_{D}\left(q^{t}\right)$. Since $F_{D}\left(q^{t}\right)$ is an increasing function of the production quantity $\left(q^{t}\right)$, this leads to a decrease in optimal production quantity value. Thus the optimal production quantity decreases over time.

(ii) We need to show that if $s^{t} \geq s^{t+1}$ and $\left(s^{t}+m-c\right)+\alpha v \beta \geq v$ then $q^{* t T} \geq q^{* t+1 T}$. So

$$
\begin{aligned}
&\left(s^{t}+m-c\right)+v \cdot \alpha \beta \geq v \\
& \Rightarrow\left(s^{t}+m-c\right) \alpha^{T-t} \beta^{T-t-1}+v \cdot \alpha^{T-t+1} \beta^{T-t} \geq v \cdot \alpha^{T-t} \beta^{T-t-1} \\
& \Rightarrow\left(s^{t}+m-c\right) \sum_{j=t+1}^{T} \alpha^{j-t} \beta^{j-t-1}+v \cdot \alpha^{T-t+1} \beta^{T-t} \\
&\left(s^{t+1}+m-c\right) \sum_{j=t+2}^{T} \alpha^{j-t-1} \beta^{j-t-2}+v \cdot \alpha^{T-t} \beta^{T-t-1} \\
& \Rightarrow \frac{c}{p+\delta\left[\left(s^{t}+m-c\right) \sum_{j=t+1}^{T} \alpha^{j-t} \beta^{j-t-1}+v \cdot \alpha^{T-t+1} \beta^{T-t}\right]} \\
& \leq c \\
& \Rightarrow \quad 1-F_{\Lambda}\left(q^{t}\right) \leq 1-F_{\Lambda}\left(q^{t+1}\right) \\
& \Rightarrow \quad q^{t} \geq q^{t+1}
\end{aligned}
$$

where the third inequality follows from $s^{t} \geq s^{t+1}$ and the fact that we are adding greater terms to the left side of inequality and smaller terms to the right side. The last step follows from the increasing property of cumulative distribution functions. This establishes (A-1) as a sufficient condition for the lemma to hold.

\section{A.4 Proof of Proposition 3}

The monopolist problem in the infinite horizon case is:

$$
\max \sum_{t=1}^{T} \alpha^{t}\left[\left(p^{t}+\delta^{t}\left(s^{t}, p^{t}\right) \frac{\left(s^{t}+m-c\right) \cdot \alpha}{1-\alpha \cdot \beta^{t}\left(s^{t}\right)}\right) \phi^{t}\left(q^{t}, p^{t}\right)-c \cdot q^{t}\right]
$$


Using the first order necessary conditions the result follows. It only remains to show that the optimal subscription price is not arbitrarily large. It suffices to show that firm's revenue increases by decreasing $s^{t}$ if $s^{t}$ is arbitrarily large. As $\lim _{s^{t} \rightarrow \infty} s^{t} \delta^{t}(\cdot)=0$ (Condition RC), the firm does not gain any subscription revenue when the subscription price is too high. Thus the revenue will be limited to the stand stales and there will be no subscription or advertisement revenue. By decreasing the subscription price, in addition to the revenue through stand sales, which will remain unaffected, the firm will also be receiving some revenue through advertisement and subscription. This shows that the optimal solution is not arbitrarily large. Thus the optimal solution is either the solution to the first order condition or $s^{t}=0$.

\section{A.5 Proof of Proposition 4}

The optimal subscription price and production quantity decision of the firm are either boundary points of the feasible region or stationary points inside the feasible region. In this setting $q^{t} \in$ $[0,+\infty)$ and $s^{t} \in[0,+\infty)$. Neither can be arbitrarily large with arguments similar to appendix A.4. So either the optimal values are 0 or they satisfy the following set of equations:

$$
\begin{aligned}
& \frac{\partial h^{t}\left(q^{t}, s^{t}\right)}{\partial s^{t}}=0 \\
& \frac{\partial h^{t}\left(q^{t}, s^{t}\right)}{\partial q^{t}}=0
\end{aligned}
$$

As shown in Proposition 3, the first equation only includes the subscription price $\left(s^{t}\right)$ decision. Thus the firm will first choose an optimal subscription price $\left(s^{t}\right)$ independent of the production quantity $\left(q^{t}\right)$ through A-3. In the next step, the optimal production quantity decision $\left(q^{t}\right)$ will be made through (A-4). In a more abstract form the objective function of firm can be viewed as:

$$
f(s, q)=f_{1}(q)+f_{2}(q) \cdot f_{3}(s)
$$

Knowing that $f_{1}(q)$ and $f_{2}(q)$ are positive values the optimal choice of $s$ to maximize $f(s, q)$ does not depend on the value of $q$.

After finding the optimal subscription price, the problem reduces to finding the optimal production quantity decision for a given subscription price, which is solved through Proposition 2.

\section{A.6 Proof of Proposition 5}

The proof for this proposition is similar to the proof of Proposition 4 and is omitted. 


\section{A.7 Proof of Proposition 6}

Given the assumptions stated in the proposition and the transformation presented in subsection 3.3.2, the firm's problem as a one-stage problem will be as follows (we have dropped the $t$ superscript for the sake of brevity):

$$
\begin{aligned}
\max \Pi(\tilde{p}, q)= & \tilde{p} E \min \{D(\tilde{p}), q\}-c q \\
\text { s.t. } & \tilde{p}, q \geq 0
\end{aligned}
$$

In the additive case we have:

$$
\begin{aligned}
\Pi(\tilde{p}, q) & =\tilde{p} E \min \{\tilde{a}-\tilde{b} \tilde{p}+\epsilon, q\}-c q=\tilde{p}\left(\int_{0}^{q-\tilde{a}+\tilde{b} \tilde{p}}(\tilde{a}-\tilde{b} \tilde{p}+\epsilon) f(\epsilon) d \epsilon+\int_{q-\tilde{a}+\tilde{b} \tilde{p}}^{1} q f(\epsilon) d \epsilon\right)-c q \\
& =-\frac{\tilde{a}^{2} \tilde{p}}{2}+\tilde{a} \tilde{b} \tilde{p}^{2}-\frac{\tilde{b}^{2} \tilde{p}^{3}}{2}-c q+\tilde{p} q+\tilde{a} \tilde{p} q-\tilde{b} \tilde{p}^{2} q-\frac{\tilde{p} q^{2}}{2}
\end{aligned}
$$

where $f(\epsilon)=1$.

Using the first order conditions, we find the optimal $\left(\tilde{p}^{*}, q^{*}\right)$ to be the solution to the system of equations (9a) and (9b). Checking the boundary conditions also ensures that the optimal solutions will be derived using the first order conditions, as $\Pi(\tilde{p}, 0)=0$ and $\Pi(\tilde{p}, \infty)=\Pi(\infty, \tilde{q})=\Pi(\infty, \infty)=$ $-\infty(\tilde{b} \neq 0)$. Thus the choice of optimal $q$ will not be on the boundary. It is also clear that $\tilde{p}<c$ (specifically $\tilde{p}=0$ ) is not an optimal solution.

The solution for the multiplicative case is along the same lines, and in that case the simplified profit function is:

$$
\begin{aligned}
\Pi(\tilde{p}, q) & =\tilde{p} E \min \left\{a\left(b_{\tilde{p}} \tilde{p}+a_{\tilde{p}}\right)^{-b} \epsilon, q\right\}-c q \\
& =\tilde{p}\left(\int_{0}^{q /\left(a\left(b_{\tilde{p}} \tilde{p}+a_{\tilde{p}}\right)^{-b}\right)} a\left(b_{\tilde{p}} \tilde{p}+a_{\tilde{p}}\right)^{-b} \epsilon f(\epsilon) d \epsilon+\int_{q /\left(a\left(b_{\tilde{p}} \tilde{p}+a_{\tilde{p}}\right)^{-b}\right)}^{1} q f(\epsilon) d \epsilon\right)-c q \\
& =\tilde{p}\left(a\left(b_{\tilde{p}} \tilde{p}+a_{\tilde{p}}\right)^{-b} * \frac{1}{2}\left(\frac{q}{a\left(b_{\tilde{p}} \tilde{p}+a_{\tilde{p}}\right)^{-b}}\right)^{2}+q\left(1-\frac{q}{a\left(b_{\tilde{p}} \tilde{p}+a_{\tilde{p}}\right)^{-b}}\right)\right)-c q \\
& =\tilde{p}\left(q-\frac{\left(b_{\tilde{p}} \tilde{p}+a_{\tilde{p}}\right)^{b}}{2 a} q^{2}\right)-c q
\end{aligned}
$$

where $f(\epsilon)=1$.

Again, using the first order conditions, the optimal $\left(\tilde{p}^{*}, q^{*}\right)$ decision is characterized as in (10b) and (10a). An argument similar to the additive case shows that the optimal virtual price is neither arbitrarily large nor on the 0 boundary. With the same argument, the optimal production quantity $(q)$ is not on the 0 boundary, and if $b_{\tilde{p}} \tilde{p}+a_{\tilde{p}}$ is positive, the optimal production quantity is not on the $\infty$ boundary either. 


\section{A.8 Proof of Proposition 7}

As shown in the proof of Proposition 6, the optimal production quantity $(q)$ solution is not on the boundaries of the feasible region $[0,+\infty)$. The optimal virtual price $(\tilde{p})$ also can not be arbitrarily large since this will lead to an arbitrarily large newsstand price $(p)$, which drives the sales to zero and yields no profit. This follows since $\lim _{p \rightarrow \infty} p \cdot D(p)=\lim _{p \rightarrow \infty} p a p^{-b} \epsilon=0$ in the multiplicative model of demand and $\lim _{p \rightarrow \infty} p \cdot D(p)=\lim _{p \rightarrow \infty} p(a-b p+\epsilon)^{+}=0$ in the additive model (recall that in the linear demand model $b \geq 0$, in the multiplicative model $a \geq 0, b \geq 1$, and in both models $\phi=\min \{D(p), q\}$ and $\epsilon$ is a bounded random variable). But it is possible that the optimal virtual price $(\tilde{p})$ results in a negative newsstand price (recall $p=b_{\tilde{p}} \tilde{p}+a_{\tilde{p}}$ ), which is not acceptable. In order to tackle this, we 1) eliminate the virtual price $(\tilde{p})$ values that result in a negative newsstand price $(p)$ and 2) add the boundary point 0 to the set of possible choices for the optimal newsstand price $(p)$ and compare it with other feasible stationary points derived from equations (10b) and (10a). This point is $\left(\tilde{p}^{t}, q^{t}\right)=\left(-a_{\tilde{p}^{t}} / b_{\tilde{p}^{t}}, \tilde{a}^{t}+1+c b_{\tilde{p}^{t}} / a_{\tilde{p}^{t}}+b a_{\tilde{p}^{t}}\right)$.

\section{A.9 Proof of Proposition 8}

The proof of this proposition is same as Proposition 1 and is omitted.

\section{A.10 Proof of Proposition 9}

We use the result in Lippman and McCardle (1997) to derive the equilibrium points. They prove that a pure-strategy Nash equilibrium exists in inventory levels $\left(q_{1}, q_{2}\right)$ in case of two newsvendors competing over quantity. This is proved using the theory of supermodular games by Topkis (1979). The following result is due to Lippman and McCardle (1997):

Characterizing Equilibrium: The pair $\left(q_{1}^{*}, q_{2}^{*}\right)$ of inventory levels is a Nash equilibrium if each firm stocks out with probability $\frac{c_{i}}{p_{i}}$ :

$$
P\left(D_{i}>q_{i}^{*}\right)=\frac{c_{i}}{p_{i}} \quad \text { for } i=1,2
$$

If the cumulative distribution of $D_{i}$ is continuous, then this condition is also necessary. Moreover, if $\gamma_{1}=\gamma_{2}=1$ (so all unsatisfied demand is reallocated), then $q_{1}^{*}+q_{2}^{*} \geq q^{*}$ : competition never leads to a decline in industry inventory.

In the above proposition, $q^{*}$ stands for the total production quantity that will maximize the sum of the profits of both firms. Using this result, the equilibrium points for the two firms when they face a uniform demand is characterized as follows. Since each firm faces the demand $D_{i}=$ 
$\Lambda_{i}+\gamma_{i}\left(\Lambda_{j}-q_{j}\right)^{+}$, the stockout probability for each of the firms is:

$$
\frac{c_{i}}{\tilde{p}_{i}}=P\left(D_{i} \geq q_{i}\right)=P\left(\Lambda_{i}+\gamma_{i}\left(\Lambda_{j}-q_{j}\right)^{+} \geq q_{i}\right)
$$

We know from the above theorem that the duopoly game has a equilibrium. We also know the necessary and sufficient condition for the equilibrium point. Utilizing this information, we focus on different sub-games of the original duopoly game and derive the equilibrium points (if any) that might exist in each sub-game. Consequently, we derive the conditions that the equilibrium point will emerge in a certain sub-game and not the other ones.

We analyze this problem in three cases by restricting domain of $q_{i}$ 's.

- Case I: $q_{i} \geq 1$ for all $i$

There is no equilibrium that will satisfy the above criteria. The above condition refers to the setting where both firms are producing more than 1 unit (the upper bound on their initial demand). Since none of them is getting any overflow of unmet demand from the other firm, each of them is facing a classic newsvendor problem and is better off by lowering its production quantity to less than 1 . Hence, this is not an equilibrium.

- Case II: $0 \leq q_{i} \leq 1$ for all $\mathrm{i}$

Assuming $0 \leq q_{i} \leq 1$ and conditioning on the value of $\Lambda_{1}$, we get

$$
\begin{aligned}
P\left(\Lambda_{1}+\gamma_{1}\left(\Lambda_{2}-q_{2}\right)^{+} \geq q_{1}\right) & =\left(1-q_{1}\right)+P\left(\gamma_{1}\left(\Lambda_{2}-q_{2}\right) \geq q_{1}-\Lambda_{1}, q_{1} \geq \Lambda_{1}\right) \\
& =\left(1-q_{1}\right)+\int_{0}^{q_{1}} P\left(\Lambda_{2} \geq \frac{q_{1}}{\gamma_{1}}+q_{2}-\frac{x}{\gamma_{1}}\right) d x \\
& =\left(1-q_{1}\right)+\int_{q_{1}+\gamma_{1} q_{2}-\gamma_{1}}^{q_{1}} P\left(\Lambda_{2} \geq \frac{q_{1}}{\gamma_{1}}+q_{2}-\frac{x}{\gamma_{1}}\right) d x \\
& =\left(1-q_{1}\right)+\int_{q_{1}+\gamma_{1} q_{2}-\gamma_{1}}^{q_{1}}\left(1-\frac{q_{1}}{\gamma_{1}}-q_{2}+\frac{x}{\gamma_{1}}\right) d x \\
& =\left(1-q_{1}\right)+\frac{\gamma_{1}\left(1-q_{2}\right)^{2}}{2}
\end{aligned}
$$

where the third equality follows from $q_{i}+\gamma_{i} q_{j}-\gamma_{i} \geq 0$ (proved below) and that $0 \leq q_{2} \leq 1$.

The same equation holds for the second firm, so we have to solve the following system of equations:

$$
\begin{aligned}
& \frac{c_{1}}{\tilde{p}_{1}}=\left(1-q_{1}\right)+\frac{\gamma_{1}\left(1-q_{2}\right)^{2}}{2} \\
& \frac{c_{2}}{\tilde{p}_{2}}=\left(1-q_{2}\right)+\frac{\gamma_{2}\left(1-q_{1}\right)^{2}}{2}
\end{aligned}
$$




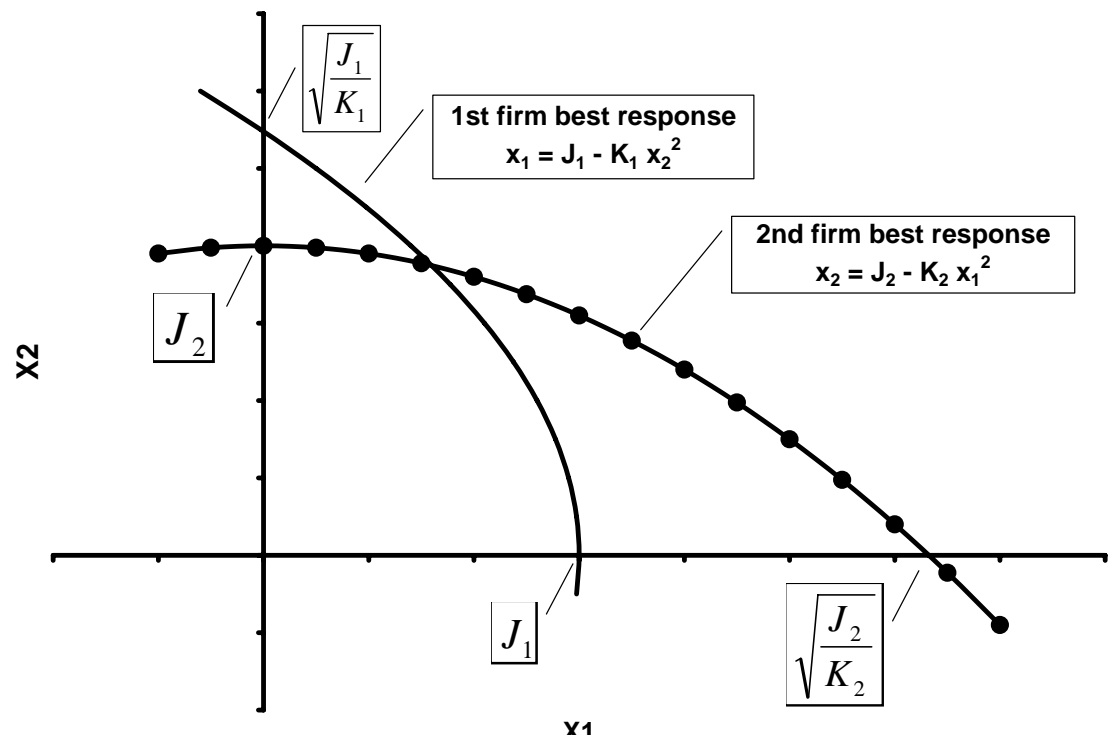

Figure 9: Best Response Functions

To analyze the general system of equations (A-6) and (A-7) look at the plot of the following system in Figure 9.

$$
\begin{aligned}
& x_{1}=J_{1}-K_{1} x_{2}^{2} \\
& x_{2}=J_{2}-K_{2} x_{1}^{2}
\end{aligned}
$$

where in our model $J_{i}=c_{i} / \tilde{p}_{i}, K_{i}=\gamma_{i} / 2$ and $x_{i}=1-q_{i}$.

In order for the above system to have a unique solution satisfying $0<q_{1}, q_{2}<1$, one of the following sets of constraints should hold:

(†) $J_{1} \leq \sqrt{\frac{J_{2}}{K_{2}}}$ and $J_{2} \leq \sqrt{\frac{J_{1}}{K_{1}}}$, or

(†) $J_{1} \geq \sqrt{\frac{J_{2}}{K_{2}}}$ and $J_{2} \geq \sqrt{\frac{J_{1}}{K_{1}}}$

First we investigate $(\dagger)$ :

$$
\begin{aligned}
& J_{2} \leq \sqrt{\frac{J_{1}}{K_{1}}} \Longrightarrow \frac{c_{2}}{\tilde{p}_{2}} \leq \sqrt{\frac{2}{\gamma_{1}}} \sqrt{\frac{c_{1}}{\tilde{p}_{1}}} \\
& J_{1} \leq \sqrt{\frac{J_{2}}{K_{2}}} \Longrightarrow \frac{c_{1}}{\tilde{p}_{1}} \leq \sqrt{\frac{2}{\gamma_{2}}} \sqrt{\frac{c_{2}}{\tilde{p}_{2}}} \Longrightarrow \frac{\gamma_{2}}{2}\left(\frac{c_{1}}{\tilde{p}_{1}}\right)^{2} \leq \frac{c_{2}}{\tilde{p}_{2}}
\end{aligned}
$$

Noting that $0 \leq \gamma_{i} \leq 1$ and $0 \leq c_{i} \leq p_{i}$, the above bounds for $c_{2} / \tilde{p}_{2}$ are consistent. So solving the equations (A-6) and (A-7) will give us the equilibrium solutions. 
Now focus on the $(\ddagger)$. Notice that:

$$
\begin{aligned}
& J_{2} \geq \sqrt{\frac{J_{1}}{K_{1}}} \Longrightarrow \frac{c_{2}}{\tilde{p}_{2}} \geq \sqrt{\frac{2}{\gamma_{1}}} \sqrt{\frac{c_{1}}{\tilde{p}_{1}}} \geq \frac{c_{1}}{\tilde{p}_{1}}(*) \\
& J_{1} \geq \sqrt{\frac{J_{2}}{K_{2}}} \Longrightarrow \frac{c_{1}}{\tilde{p}_{1}} \geq \sqrt{\frac{2}{\gamma_{2}}} \sqrt{\frac{c_{2}}{\tilde{p}_{2}}} \geq \frac{c_{2}}{\tilde{p}_{2}} \stackrel{*}{\Longrightarrow} \frac{c_{1}}{\tilde{p}_{1}}=\frac{c_{2}}{\tilde{p}_{2}}=\frac{c}{\tilde{p}}
\end{aligned}
$$

So we will have:

$$
\frac{c}{\tilde{p}} \geq \sqrt{\frac{2}{\gamma_{1}}} \sqrt{\frac{c}{\tilde{p}}} \Rightarrow \sqrt{\frac{c}{\tilde{p}}} \geq \sqrt{\frac{2}{\gamma_{1}}} \Rightarrow \gamma_{1} \geq \frac{2 \tilde{p}}{c} \geq 1
$$

where the last inequality follows from the fact that virtual price is higher than the cost. But by definition $\gamma_{1}$ is less than 1 . Hence, the second set of constraints will never hold with our assumptions.

It remains to show that $q_{i}+\gamma_{i} q_{j}-\gamma_{i} \geq 0$. Define $w_{1}$ as the solution to $P\left(\Lambda_{1} \geq w_{1}\right)=\sigma_{1}$. Recall $\Lambda_{1}$ is a Uniform $[0,1]$ random variable. Therefore, $w_{1}=1-\sigma_{1}$. Compare $w_{1}$ with $q_{1}$, where $q_{1}$ is the solution to $P\left(D_{1} \geq q_{1}\right)=\sigma_{1}$. Recall $D_{1}=\Lambda_{1}+\gamma_{1}\left(\Lambda_{2}-q_{2}\right)^{+}$. Therefore $q_{1} \geq w_{1}$. Similarly we can prove $q_{2} \geq w_{2}$ (where $w_{2}$ is defined analogous to $w_{1}$ ). So

$$
q_{1} / \gamma_{1}+q_{2} \geq\left(1-\sigma_{1}\right) / \gamma_{1}+1-\sigma_{2} \geq \sigma_{2}+1-\sigma_{2}=1
$$

where the second inequality follows from the assumption on the bounds of $\gamma_{i}$ 's.

- Case III: $1 \leq q_{i}$ and $1 \geq q_{-i}$

This is the setting where one of the firms is producing more than 1 unit. Without loss of generality, assume $q_{1}>1$ and $q_{2}<1$.

In this case, because there is no overflow of demand from firm 1 to firm 2, the second firm will be solving a classic newsvendor problem, and its optimal quantity decision will be:

$$
q_{2}^{*}=1-\frac{c_{2}}{\tilde{p}_{2}}
$$

Knowing this, the first firm's demand function will be

$$
D_{1}=\Lambda_{1}+\gamma_{1}\left(\Lambda_{2}-1+\frac{c_{2}}{\tilde{p}_{2}}\right)^{+}
$$

Now, the first firm is also facing a classic newsvendor problem and we just need to specify distribution of $D_{1}$. This distribution is derived through Lemma 3.

Lemma 3 Distribution of sum of two independent random variables $X_{1}=U$ nif $[0,1]$ and $X_{2}=$ Unif $[0, \alpha], \alpha<1$ is 
1. $0 \leq b \leq \alpha: P\left(X_{1}+X_{2} \leq b\right)=b^{2} / 2 \alpha$

2. $\alpha \leq b \leq 1$ : $P\left(X_{1}+X_{2} \leq b\right)=(2 b-\alpha) / 2$

3. $1 \leq b \leq 1+\alpha$ : $P\left(X_{1}+X_{2} \leq b\right)=-(b-\alpha-1)(b-\alpha+1) / 2 \alpha+(b-1) / \alpha$

Proof: The proof is straightforward and omitted.

$D_{1}$ is sum of two random variables: one of them is a uniform $[0,1]$, and the other one is a random variable which has a mass probability $1-c_{2} / \tilde{p}_{2}$ on 0 and the rest is uniformly distributed on the interval $\left[0, \gamma_{1} c_{2} / \tilde{p}_{2}\right]$. Assume $\Lambda_{3}$ is a random variable which is uniformly distributed on $\left[0, \gamma_{1} c_{2} / \tilde{p}_{2}\right]$. So the new random variable will have the following distribution:

$$
\begin{aligned}
P\left(D_{1} \leq a\right) & =P\left(\Lambda_{1} \leq a, \Lambda_{2} \leq q_{2}^{*}\right)+P\left(\Lambda_{1}+\Lambda_{3} \leq a, \Lambda_{2} \geq q_{2}^{*}\right) \\
& =P\left(\Lambda_{1} \leq a\right)\left(1-\frac{c_{2}}{\tilde{p}_{2}}\right)+P\left(\Lambda_{1}+\Lambda_{3} \leq a\right)\left(\frac{c_{2}}{\tilde{p}_{2}}\right)
\end{aligned}
$$

Using the Lemma 3, we can write the probability distribution of $D_{1}$.

1. $0 \leq a \leq \gamma_{1} c_{2} / \tilde{p}_{2}$

$$
\begin{aligned}
P\left(D_{1} \leq a\right) & =a\left(1-\frac{c_{2}}{\tilde{p}_{2}}\right)+P\left(\Lambda_{1}+\Lambda_{3} \leq a\right)\left(\frac{c_{2}}{\tilde{p}_{2}}\right) \\
& =a\left(1-\frac{c_{2}}{\tilde{p}_{2}}\right)+a^{2} /\left(2 \gamma_{1} \frac{c_{2}}{\tilde{p}_{2}}\right) \cdot\left(\frac{c_{2}}{\tilde{p}_{2}}\right)=a\left(1-\frac{c_{2}}{\tilde{p}_{2}}\right)+\frac{a^{2}}{2 \gamma_{1}}
\end{aligned}
$$

2. $\gamma_{1} c_{2} / \tilde{p}_{2} \leq a \leq 1$

$$
\begin{aligned}
P\left(D_{1} \leq a\right) & =a\left(1-\frac{c_{2}}{\tilde{p}_{2}}\right)+P\left(\Lambda_{1}+\Lambda_{3} \leq a\right)\left(\frac{c_{2}}{\tilde{p}_{2}}\right) \\
& =a\left(1-\frac{c_{2}}{\tilde{p}_{2}}\right)+\left(2 a-\gamma_{1} \frac{c_{2}}{\tilde{p}_{2}}\right) / 2 \cdot\left(\frac{c_{2}}{\tilde{p}_{2}}\right)=a-\frac{\gamma_{1}}{2}\left(\frac{c_{2}}{\tilde{p}_{2}}\right)^{2}
\end{aligned}
$$

3. $1 \leq a \leq 1+\gamma_{1} c_{2} / \tilde{p}_{2}$

$$
\begin{aligned}
P\left(D_{1} \leq a\right) & =\left(1-\frac{c_{2}}{\tilde{p}_{2}}\right)+P\left(\Lambda_{1}+\Lambda_{3} \leq a\right)\left(\frac{c_{2}}{\tilde{p}_{2}}\right) \\
& =\left(1-\frac{c_{2}}{\tilde{p}_{2}}\right)+\left[-1 /\left(2 \gamma_{1} \frac{c_{2}}{\tilde{p}_{2}}\right) \cdot\left(a-\gamma_{1} \frac{c_{2}}{\tilde{p}_{2}}-1\right)\left(a-\gamma_{1} \frac{c_{2}}{\tilde{p}_{2}}+1\right)+1 /\left(\gamma_{1} \frac{c_{2}}{\tilde{p}_{2}}\right)(a-1)\right]\left(\frac{c_{2}}{\tilde{p}_{2}}\right) \\
& =\left(1-\frac{c_{2}}{\tilde{p}_{2}}\right)+\left[-1 /\left(2 \gamma_{1}\right) \cdot\left(a-\gamma_{1} \frac{c_{2}}{\tilde{p}_{2}}-1\right)\left(a-\gamma_{1} \frac{c_{2}}{\tilde{p}_{2}}+1\right)+\frac{1}{\gamma_{1}}(a-1)\right] \\
& =1-\frac{c_{2}}{\tilde{p}_{2}}-\frac{a^{2}}{2 \gamma_{1}}+\frac{a c_{2}}{\tilde{p}_{2}}-\frac{1}{2} \frac{\gamma_{1} c_{2}^{2}}{\tilde{p}_{2}^{2}}-\frac{1}{2 \gamma_{1}}+\frac{a}{\gamma_{1}}
\end{aligned}
$$

Now, the solution to the newsvendor problem of the first firm will be $P\left(D_{1} \geq q_{1}^{*}\right)=c_{1} / \tilde{p}_{1}$. We are looking for the equilibrium point in the sub-game where $q_{1}^{*}$ is greater than 1 (otherwise the second 
firm's problem would not have been in the form we assumed), hence we have to use the third case. So $q_{1}^{*}$ will be the solution to:

$$
\begin{gathered}
\frac{c_{2}}{\tilde{p}_{2}}+\frac{q_{1}^{* 2}}{2 \gamma_{1}}-\left(\frac{1}{\gamma_{1}}+\frac{c_{2}}{\tilde{p}_{2}}\right) q_{1}^{*}+\frac{1}{2} \frac{\gamma_{1} c_{2}^{2}}{\tilde{p}_{2}^{2}}+\frac{1}{2 \gamma_{1}}=\frac{c_{1}}{\tilde{p}_{1}} \Rightarrow \\
f\left(q_{1}^{*}\right):=\frac{1}{2 \gamma_{1}} q_{1}^{* 2}-\left(\frac{1}{\gamma_{1}}+\frac{c_{2}}{\tilde{p}_{2}}\right) q_{1}^{*}+\frac{1}{2} \frac{\gamma_{1} c_{2}^{2}}{\tilde{p}_{2}^{2}}+\frac{1}{2 \gamma_{1}}+\frac{c_{2}}{\tilde{p}_{2}}-\frac{c_{1}}{\tilde{p}_{1}}=0
\end{gathered}
$$

It remains to investigate when the above equation has an answer. For that, we check the discriminant of the quadratic equation.

$$
\Delta=\left[-1\left(\frac{1}{\gamma_{1}}+\frac{c_{2}}{\tilde{p}_{2}}\right)\right]^{2}-4\left(\frac{1}{2 \gamma_{1}}\right)\left(\frac{1}{2} \frac{\gamma_{1} c_{2}^{2}}{\tilde{p}_{2}^{2}}+\frac{1}{2 \gamma_{1}}+\frac{c_{2}}{\tilde{p}_{2}}-\frac{c_{1}}{\tilde{p}_{1}}\right)=\frac{2 c_{1}}{\tilde{p}_{1}} \geq 0
$$

So there is always a solution. In order to make sure this solution is in $[0,1]$ interval, we investigate the value of the quadratic polynomial in its extreme points.

$$
f\left(q_{1}^{*}=1\right)=\frac{\gamma_{1}}{2}\left(\frac{c_{2}}{\tilde{p}_{2}}\right)^{2}-\frac{c_{1}}{\tilde{p}_{1}}=\frac{\gamma_{1}}{2} \sigma_{2}^{2}-\sigma_{1}, \quad f\left(q_{1}^{*}=1+\gamma_{1} c_{2} / \tilde{p}_{2}\right)=-\frac{c_{1}}{\tilde{p}_{1}} \leq 0
$$

Find the condition where $f\left(q_{1}^{*}=1\right) \geq 0$. Solving this inequality gives us the condition $\sigma_{2} \geq$ $\sqrt{2 \sigma_{1} / \gamma_{1}}$, which is the reverse of the condition for the previous case of duopoly game (symmetric equilibrium condition). Hence, if this condition is met, the asymmetric equilibrium with firm 1 producing more than 1 unit will emerge. The analogous case will be when firm 2 produces more than 1 unit and is not shown here for brevity. As shown in the proof of the symmetric equilibrium, it is not possible to have $\sigma_{1} \geq \sqrt{2 \sigma_{2} / \gamma_{2}}$ and $\sigma_{2} \geq \sqrt{2 \sigma_{1} / \gamma_{1}}$ at the same time.

\section{A.11 Proof of Lemma 2}

Any choice of $\left(c_{1}, p_{1}\right)$ and $\left(c_{2}, p_{2}\right)$ will result in two unique values for $\sigma_{1}$ and $\sigma_{2}$. Thus one can find the maximum $\gamma_{i}^{*}$ 's such that $\sigma_{i} \leq \sqrt{2 \sigma_{-i} / \gamma_{-i}^{*}}$ and $\gamma_{i} \leq\left(1-\sigma_{i}\right) / \sigma_{-i}$ hold. Choose $\gamma^{*}=\min _{i}\left\{\gamma_{i}^{*}\right\}$, and the result follows from Proposition 9.

\section{A.12 Proof of Proposition 10}

From (13a) and (13b), we are solving a system of the following form:

$$
\begin{gathered}
x_{1}=k_{1}-c_{1} x_{2}^{2} \\
x_{2}=k_{2}-c_{2} x_{1}^{2}
\end{gathered}
$$

This system of equations results in

$$
\begin{aligned}
& x_{1} \leq k_{1}:=u_{1}^{(0)} \\
& x_{2} \leq k_{2}:=u_{2}^{(0)}
\end{aligned}
$$


We also know that $x_{1} \geq 0=l_{1}^{(0)}$ and $x_{2} \geq 0=l_{2}^{(0)}$. Now we show a round-robin scheme converges for the above system.

$$
\begin{aligned}
& x_{1} \leq u_{1}^{(k)} \Rightarrow-x_{1}^{2} \geq-u_{1}^{(k)^{2}} \stackrel{(I I)}{\Rightarrow} x_{2} \geq k_{2}-c_{2} u_{1}^{(k)^{2}}:=l_{2}^{(k+1)} \\
& x_{1} \geq l_{1}^{(k)} \Rightarrow-x_{1}^{2} \leq-l_{1}^{(k)^{2}} \stackrel{(I I)}{\Rightarrow} x_{2} \leq k_{2}-c_{2} l_{1}^{(k)^{2}}:=u_{2}^{(k+1)}
\end{aligned}
$$

Similarly $x_{1} \geq k_{1}-c_{1} u_{2}^{(k)^{2}}:=l_{1}^{(k+1)}$ and $x_{1} \leq k_{1}-c_{1} l_{2}^{(k)^{2}}:=u_{1}^{(k+1)}$.

Repeating the above iteration results in

$$
\begin{aligned}
& x_{1} \geq k_{1}-c_{1} u_{2}^{(k+1)^{2}}:=l_{1}^{(k+2)} \\
& x_{1} \leq k_{1}-c_{1} l_{2}^{(k+1)^{2}}:=u_{1}^{(k+2)}
\end{aligned}
$$

So we will have

$$
\begin{aligned}
u_{1}^{(k+2)}-l_{1}^{(k+2)} & =\left(k_{1}-c_{1} l_{2}^{(k+1)^{2}}\right)-\left(k_{1}-c_{1} u_{2}^{(k+1)^{2}}\right)=c_{1}\left(u_{2}^{(k+1)^{2}}-l_{2}^{(k+1)^{2}}\right) \\
& =c_{1}\left(u_{2}^{(k+1)}+l_{2}^{(k+1)}\right)\left(u_{2}^{(k+1)}-l_{2}^{(k+1)}\right) \\
& \stackrel{(\aleph)}{=} c_{1}\left(u_{2}^{(k+1)}+l_{2}^{(k+1)}\right)\left(k_{2}-c_{2} l_{1}^{(k)^{2}}-k_{2}+c_{2} u_{1}^{(k)^{2}}\right) \\
& =c_{1}\left(u_{2}^{(k+1)}+l_{2}^{(k+1)}\right) c_{2}\left(u_{1}^{(k)}+l_{1}^{(k)}\right)\left(u_{1}^{(k)}-l_{1}^{(k)}\right)
\end{aligned}
$$

Notice that $u_{1}^{(k)}+l_{1}^{(k)}$ is always strictly less than 2 (since in our setting $u_{i}^{(0)}=k_{i}=\sigma_{i} \leq 1$ ). If it is equal to 2 , then we need $u_{1}^{(k)}=l_{1}^{(k)}=1$. But this can only happen if $u_{1}^{(k-1)}=l_{1}^{(k-1)}=1$ and so on. Since we never start from $u_{1}^{(0)}=l_{1}^{(0)}=1$, this will never happen.

In our problem $c_{i}=\gamma_{i} / 2$ and $\gamma_{i}, l_{i}^{k}, u_{i}^{k} \in[0,1]$, so the multiplier of $\left(u_{1}^{(k)}-l_{1}^{(k)}\right)$ in the above equation is between 0 and 1 . Thus, we have established that $u_{1}^{(k+2)}-l_{1}^{(k+2)}=m\left(u_{1}^{(k)}-l_{1}^{(k)}\right)$ where $m \in[0,1]$. Considering the above note on $u_{1}^{(k)}+l_{1}^{(k)}$ being strictly less than $2, m$ is strictly less than 1 . This shows that a round-robin scheme to find the solution of the above system converges linearly.

\section{A.13 Proof of Proposition 11}

The proof is similar to the proof the monopolist case and is omitted here. It is important to notice that the same proof holds since the choice of each firm's optimal subscription price is independent of its optimal production quantity and the decisions of the other firm as noted in equation (15).

\section{A.14 Proof of Proposition 12}

The proof is similar to the proof of the monopolist case and is omitted here. Each firm can choose the optimal subscription price through Proposition 11 independent of the optimal production quantity decision, and then the problem reduces to the quantity game studied in Proposition 9. 